\title{
TOTALLY INVARIANT DIVISORS OF INT-AMPLIFIED ENDOMORPHISMS OF NORMAL PROJECTIVE VARIETIES
}

\author{
GUOLEI ZHONG
}

\begin{abstract}
We consider an arbitrary int-amplified surjective endomorphism $f$ of a normal projective variety $X$ over $\mathbb{C}$ and its $f^{-1}$-stable prime divisors. We extend the early result in [32, Theorem 1.3] for the case of polarized endomorphisms to the case of intamplified endomorphisms.

Assume further that $X$ has at worst Kawamata log terminal singularities. We prove that the total number of $f^{-1}$-stable prime divisors has an optimal upper bound $\operatorname{dim} X+$ $\rho(X)$, where $\rho(X)$ is the Picard number. Also, we give a sufficient condition for $X$ to be rationally connected and simply connected. Finally, by running the minimal model program (MMP), we prove that, under some extra conditions, the end product of the MMP can only be an elliptic curve or a single point.
\end{abstract}

\section{CONTEnTs}

1. Introduction 1

2. Preliminaries 3

2.1. Notation and terminology 3

2.2. Int-amplified endomorphisms 4

2.3. Rational connectedness of varieties 6

2.4. Properties for polarized cases 7

3. Proof of Theorem $1.1 \quad 13$

3.1. The Case $\operatorname{dim} X=1 \quad 13$

3.2. The Case $\operatorname{dim} X \geq 2 \quad 14$

References $\quad 27$

\section{INTRODUCTION}

We work over the complex numbers field $\mathbb{C}$. This article generalizes the early result of [32, Theorem 1.3]. We extend the result for the case of polarized endomorphisms to the case of int-amplified endomorphisms. The main method we use is to run the minimal

2010 Mathematics Subject Classification. 14E30, 32H50, 08A35,

Key words and phrases. int-amplified endomorphism, minimal model program, rationally connected variety. 
model program (MMP) equivariantly so that we can reduce the dimension of varieties and then use the induction. It is accessible under some good conditions for the variety $X$ (cf. [22, Theorem 1.10]). See Theorem 2.3 for a detailed description.

Suppose $X$ is a projective variety. Let $f: X \rightarrow X$ be a surjective endomorphism. The endomorphism $f$ is said to be int-amplified, if there exist ample Cartier divisors $H$ and $L$ such that $f^{*} L-L=H$. A prime divisor $V$ on $X$ is said to be totally invariant under the endomorphism $f$, if $f^{-1}(V)=V$ set-theoretically. To simplify the expression, we first introduce a notation for the set of totally invariant prime divisors for the int-amplified endomorphism $f: X \rightarrow X$ :

$$
\mathrm{TI}_{f}(X)=\left\{V \mid V \text { is a prime divisor on } X \text { such that } f^{-1}(V)=V\right\} .
$$

In this article, we will bound the cardinality $c:=\# \mathrm{TI}_{f}(X)$. It turns out that there exists an optimal upper bound which is determined by the Picard number and dimension of $X$. Also, we want to give a sufficient condition for $\left.f^{*}\right|_{\operatorname{Pic}(X)}$ to be diagonalizable over $\mathbb{Q}$ by applying some early results. Moreover, under some extra conditions, we show that the variety $X$ is rationally connected and simply connected with respect to complex topology.

The following is our main result.

Theorem 1.1. Let $X$ be a projective variety of dimension $n$ with only $\mathbb{Q}$-factorial Kawamata log terminal singularities, and $f: X \rightarrow X$ an int-amplified endomorphism. Let $V_{j}(1 \leq j \leq c)$ be all the prime divisors in $\mathrm{TI}_{f}(X)$. Then we have (with $\left.\rho:=\rho(X)\right)$ :

(1) $c \leq n+\rho$. Furthermore, if $c \geq 1$, then the pair $\left(X, \sum V_{j}\right)$ is $\log$ canonical and $X$ is uniruled.

(2) Suppose $c \geq n+\rho-2$. Then either $X$ is rationally connected and simply connected with respect to complex topology, or there is a fibration $X \rightarrow E$ onto an elliptic curve $E$ such that every fibre is normal, irreducible, equi-dimensional and rationally connected. Further, in the latter case, for some integer $t \geq 1, f^{t}$ descends to an int-amplified endomorphism $g_{E}: E \rightarrow E$. In both cases, $\left.\left(f^{t}\right)^{*}\right|_{\mathrm{NS}_{\mathbb{Q}}(X)}$ is diagonalizable over $\mathbb{Q}$.

(3) Suppose $c \geq n+\rho-1$. Then $X$ is rationally connected and simply connected with respect to complex topology. Further, for some integer $t \geq 1,\left.\left(f^{t}\right)^{*}\right|_{\operatorname{Pic}(X)}$ is diagonalizable over $\mathbb{Q}$.

(4) Suppose $c \geq n+\rho$. Then $c=n+\rho, K_{X}+\sum_{j=1}^{n+\rho} V_{j} \sim_{\mathbb{Q}} 0$ and $f$ is étale outside $\left(\bigcup V_{j}\right) \cup f^{-1}(\operatorname{Sing} X)$.

One direct result of our main theorem is as follows. We refer to [3] for the toric pair.

Corollary 1.2. Suppose $X$ is a normal projective variety with only $\mathbb{Q}$-factorial Kawamata log terminal singularities, and $f: X \rightarrow X$ is an int-amplified endomorphism of $X$. If 
the cardinality c of $\mathrm{TI}_{f}(X)$ satisfies $c=\rho(X)+\operatorname{dim} X$ (achieving the upper bound) as in Theorem 1.1 (4), then $\left(X, \sum V_{j}\right)$ is a toric pair.

Indeed, Corollary 1.2 follows immediately from [3, Theorem 1.2] and our main theorem, in which case, the complexity (cf. [3, Definition 1.1]) is zero. So $\left(X, \sum V_{j}\right)$ is a toric pair.

Let $\mathrm{N}^{1}(X)=\mathrm{NS}(X) \otimes_{\mathbb{Z}} \mathbb{R}$. Comparing with the case of polarized endomorphisms, we remove the hypothesis (cf. [32, Theorem 1.3]): either $\left.f^{*}\right|_{\mathrm{N}^{1}(X)}=q \mathrm{id}_{\mathrm{N}^{1}(X)}$, or $n \leq 3$. In general, $\left.f^{*}\right|_{\mathrm{N}^{1}(X)}$ may not be a scalar matrix even in the case of polarized endomorphisms (cf. [23, Example 7.1]). In our case of int-amplified endomorphisms, the diagonalizable result holds even when $c=\rho(X)+\operatorname{dim} X-1$, extending [32, Theorem 1.3]. Moreover, we add more details to the proof of [32, Theorem 1.3 and Proposition 2.12].

For the organization of the paper, we begin with several preliminaries in Section 2. Then we give a detailed proof for our Theorem 1.1 in Section 3.

Acknowledgments. The author would like to deeply thank Professor De-Qi Zhang for many inspiring ideas and discussions. Also, he would like to thank Doctor Sheng Meng for the result of running MMP [22] on int-amplified endomorphisms, and the referees for many constructive suggestions to improve the paper.

\section{Preliminaries}

2.1. Notation and terminology. Let $X$ be a $\mathbb{Q}$-factorial normal projective variety of dimension $n$ over $\mathbb{C}$. Let $D$ be a Cartier divisor on $X$, and $\mathcal{O}_{X}(D)$ its corresponding invertible sheaf. We often identify $D$ with $\mathcal{O}_{X}(D)$. Since $X$ is $\mathbb{Q}$-factorial, any Weil divisor $B$ on $X$ is $\mathbb{Q}$-Cartier, i.e. there exists some integer $m>0$, such that $m B$ is Cartier. We use $K_{X}$ to denote a canonical divisor of the variety $X$.

In correspondence with notations in [10], let $\mathrm{NS}(X)=\operatorname{Pic}(X) / \operatorname{Pic}^{\circ}(X)$ denote the Néron-Severi group of $X$. Let $\mathrm{NS}_{\mathbb{Q}}(X):=\mathrm{NS}(X) \otimes_{\mathbb{Z}} \mathbb{Q}$ and $\mathrm{N}^{1}(X):=\mathrm{NS}(X) \otimes_{\mathbb{Z}} \mathbb{R}$. Denote by $q(X)$ the irregularity $h^{1}\left(X, \mathcal{O}_{X}\right):=\operatorname{dim} \mathrm{H}^{1}\left(X, \mathcal{O}_{X}\right)$.

Let $\mathrm{N}_{n-1}(X)$ denote the space of weakly numerically equivalent classes of Weil $\mathbb{R}$ divisors (cf. [23, Definition 2.2]). When $X$ is normal, we can regard $\mathrm{N}^{1}(X)$ as a subspace of $\mathrm{N}_{n-1}(X)$ (cf. [33, Lemma 3.2]).

Let $f: X \rightarrow X$ be a finite surjective endomorphism. We can define the pullback of $r$-cycles for $f$, such that $f^{*}$ induces an automorphism of $\mathrm{N}_{r}(X)$ and $f_{*} f^{*}=(\operatorname{deg} f)$ id. More precisely, the pullback $f^{*}: \mathrm{N}_{r}(X) \rightarrow \mathrm{N}_{r}(X)$ is defined by $f^{*}=(\operatorname{deg} f)\left(f_{*}\right)^{-1}$. We recall the following cones, which are $\left(f^{*}\right)^{ \pm}$-invariant (cf. [23, Definition 2.4]).

- $\operatorname{Amp}(X)$ : the set of classes of ample $\mathbb{R}$-Cartier divisors in $\mathrm{N}^{1}(X)$;

- $\operatorname{Nef}(X)$ : the set of classes of nef $\mathbb{R}$-Cartier divisors in $\mathrm{N}^{1}(X)$; 
- $\mathrm{PE}(X)$ : the closure of the set of classes of effective $(n-1)$-cycles with $\mathbb{R}$-coefficients in $\mathrm{N}_{n-1}(X)$.

The Theorem of the Base of Néron-Severi asserts that the real space $\mathrm{N}_{1}(X)$ of 1-cycles with real coefficients modulo numerical equivalence is a finite dimensional $\mathbb{R}$-vector space. There is a natural perfect pairing:

$$
\mathrm{N}_{1}(X) \times \mathrm{N}^{1}(X) \rightarrow \mathbb{R}
$$

Then $\operatorname{rank}_{\mathbb{R}} \mathrm{N}^{1}(X)=\operatorname{rank}_{\mathbb{R}} \mathrm{N}_{1}(X)=\rho(X)$, the Picard number of $X$.

We use the symbol $\sim\left(\right.$ resp. $\sim_{\mathbb{Q}}, \equiv$ or $\equiv_{w}$ ) to denote the linear (resp. $\mathbb{Q}$-linear, numerical or weak numerical) equivalence relation. By [1, CH. XIII, Theorem 4.6]), we see that numerically equivalent divisors are algebraically equivalent up to a positive multiple.

We refer to [26] for the definition and general properties of a pseudo-effective Weil divisor on $X$. Also, we refer to [21, CH.2] for the definition and general properties of Kodaira dimension and Iitaka dimension of any line bundle over $X$.

In addition, we now assume that $X$ has at worst Kawamata log terminal (klt) singularities. We refer to [17, Definitions 2.28 and 2.34] for the definition of discrepancies and different kinds of singularities. The next proposition about the inversion of adjunction (cf. [13, Theorem 1]) is useful for our proof.

Proposition 2.1 (cf. [13]). Let $(X, S+B)$ be a log pair (i.e. $K_{X}+S+B$ is $\mathbb{Q}$-Cartier and all the coefficients of irreducible components of $S+B$ are in $[0,1])$ such that $S$ is a reduced divisor which has no common component with the support of $B$, let $\nu: S^{\nu} \rightarrow X$ denote the normalization of $S$, and let $B^{\nu}$ denote the different of $B$ on $S^{\nu}$ (so that $\left.K_{S^{\nu}}+B^{\nu}=\nu^{*}\left(K_{X}+S+B\right)\right)$. Then $(X, S+B)$ is log canonical near $S$ if and only if $\left(S^{\nu}, B^{\nu}\right)$ is $\log$ canonical.

Suppose $f: X \rightarrow X$ is a finite surjective endomorphism of a normal variety $X$. Since $X$ is normal, it is regular in codimension one. Then one can define the pullback of a Weil divisor $H$ on $X$, as the closure of $\left(\left.f\right|_{U}\right)^{*}\left(\left.H\right|_{U}\right)$ where $U \subseteq X$ is a smooth open locus on $X$ and $X \backslash U$ is a codimension $\geq 2$ closed subset. Furthermore, when $H$ is $\mathbb{Q}$-Cartier, the pullback we discussed above coincides with the usual pullback of $\mathbb{Q}$-Cartier divisor.

2.2. Int-amplified endomorphisms. In this subsection, we first recall the definitions of polarized, amplified and int-amplified endomorphisms. Then we refer to [22] for the general properties of int-amplified endomorphisms.

Definition 2.2. Let $f: X \rightarrow X$ be a surjective endomorphism of a projective variety $X$. We say that

(1) $f$ is polarized if $f^{*} D \sim q D$ for some ample Cartier divisor $D$ and integer $q>1$; 
(2) $f$ is amplified if $f^{*} D-D=H$ for some Cartier divisor $D$ and ample Cartier divisor $H$; and

(3) $f$ is int-amplified if $f^{*} D-D=H$ for some ample Cartier divisors $D$ and $H$.

It follows from the definition that $(1) \Rightarrow(3) \Rightarrow(2)$. One can also check directly that if $f$ is an int-amplified endomorphism, then any power of $f$ is also int-amplified.

In what follows, we will recall the theorem below (cf. [22, Theorem 1.10]), which ensures that we can run equivariant MMP and then do the induction on the dimension of $X$. It extends the result of equivariant MMP (cf. [23, Theorem 1.8]) for the case of polarized endomorphisms. Recall that a normal projective variety $X$ is said to be $Q$-abelian if there exists a finite surjective morphism $A \rightarrow X$ étale in codimension one (or quasi-étale in short) with $A$ an abelian variety.

Theorem 2.3 (cf. [22]). Let $f: X \rightarrow X$ be an int-amplified endomorphism of a $\mathbb{Q}$ factorial Kawamata log terminal projective variety $X$. Then replacing $f$ by a positive power, there exist a Q-abelian variety $Y$, a morphism $X \rightarrow Y$, and an $f$-equivariant relative minimal model program over $Y$

$$
X=X_{0}-->X_{1}-->\cdots-->X_{i}-->\cdots-->X_{l}-->X_{l+1}=Y
$$

which means a positive power of $f$ descends to an endomorphism $g_{i}$ on each $X_{i}$, for $1 \leq i \leq l+1$, with every $u_{i}: X_{i} \rightarrow X_{i+1}$ a divisorial contraction, a flip or a Fano contraction over $Y$, of a $K_{X_{i}}$-negative extremal ray. Further, we have:

(1) If $K_{X}$ is pseudo-effective, then $X=Y$ and it is Q-abelian.

(2) If $K_{X}$ is not pseudo-effective, then for each $i, X_{i} \rightarrow Y$ is equi-dimensional and holomorphic with every fibre (irreducible) rationally connected and $g_{i}$ is intamplified. The last rational map $X_{l} \rightarrow X_{l+1}=Y$ is a Fano contraction (a morphism).

(3) $\left.f^{*}\right|_{\mathrm{N}^{1}(X)}$ is diagonalizable over $\mathbb{C}$ if and only if so is $\left.g_{l+1}^{*}\right|_{\mathrm{N}^{1}(Y)}$.

To make our symbols symmetric, we may denote by $g_{0}$ some power $f^{t}$, so that $g_{0}$ descends to the int-amplified endomorphism $g_{i}$ of $X_{i}$ for each $i$.

Remark 2.4. With the same symbols given above, we shall prove later that, for a divisorial contraction or a flip $X_{i} \rightarrow X_{i+1}$, we have the equality $\# \mathrm{TI}_{g_{i+1}}\left(X_{i+1}\right)=\# \operatorname{TI}_{g_{i}}\left(X_{i}\right)-$ $\left(\rho\left(X_{i}\right)-\rho\left(X_{i+1}\right)\right)$ (cf. Lemma 3.4), while for a Mori fibre contraction $X_{r} \rightarrow X_{r+1}$, the inequality $\# \mathrm{TI}_{g_{r+1}}\left(X_{r+1}\right) \geq \# \mathrm{TI}_{g_{r}}\left(X_{r}\right)-\left(\operatorname{dim} X_{r}-\operatorname{dim} X_{r+1}\right)-1$ holds (cf. Lemma 3.7$)$. Here, $\# \mathrm{TI}_{g_{i}}\left(X_{i}\right)$ denotes the cardinality of the set $\mathrm{TI}_{g_{i}}\left(X_{i}\right)$ of totally invariant divisors for the int-amplified endomorphism $g_{i}: X_{i} \rightarrow X_{i}$. 
When proving Theorem 2.3, the following statements for the case of int-amplified endomorphisms are useful. Lemma 2.5 is a special case of [22, Lemma 3.5] and Lemma 2.6 was proved in [22, Theorem 3.3].

Lemma 2.5 (cf. [22]). Let $\pi: X \rightarrow Y$ be a generically finite and surjective morphism of projective varieties. Suppose $f: X \rightarrow X$ and $g: Y \rightarrow Y$ are two surjective endomorphisms such that $g \circ \pi=\pi \circ f$. Then $f$ is int-amplified if and only if so is $g$.

Lemma 2.6 (cf. [22]). Let $f: X \rightarrow X$ be an int-amplified surjective endomorphism of a projective variety $X$. Then all the eigenvalues of $\left.f^{*}\right|_{\mathrm{N}^{1}(X)}$ are of modulus greater than 1 .

If $X$ admits an int-amplified endomorphism $f$, then for any $D \in \mathrm{TI}_{f}(X), f^{*} D=s D$ for some integer $s>1$. Further, we have $\operatorname{deg} f>1$ (cf. [22, Lemma 3.7]).

An amplified morphism was first defined by Krieger and Reschke (cf. [20]), and Fakhruddin showed the following very motivating result in [7, Theorem 5.1]. A subset $Z \subseteq X$ is said to be $f$-periodic if $f^{s}(Z)=Z$ for some $s>0$. Lemma 2.7 says that $f$-periodic points are dense if $f$ is amplified.

Lemma 2.7 (cf. [7]). Let $X$ be a projective variety over an algebraically closed field $k$. Suppose $f: X \rightarrow X$ is a dominant morphism and $\mathcal{L}$ a line bundle on $X$ such that $f^{*} \mathcal{L} \otimes \mathcal{L}^{-1}$ is ample. Then the subset $X(k)$ consisting of periodic points of $f$ is Zariski dense in $X$.

2.3. Rational connectedness of varieties. We refer to [18, CH. IV, Definitions 1.1 and 3.2] for the definitions of uniruled varieties and rationally connected varieties. We recall that a complete variety $X$ is $\log Q$-Fano, if there exists an effective $\mathbb{Q}$-divisor $D$ such that the pair $(X, D)$ is klt and $-\left(K_{X}+D\right)$ is an ample $\mathbb{Q}$-Cartier divisor.

Remark 2.8. If $X$ is a $\mathbb{Q}$-Gorenstein normal projective variety over an algebraically closed field of characteristic zero with the canonical divisor $K_{X}$ not pseudo-effective, then $X$ is uniruled (cf. [2, 0.3 Corollary]).

Next, we review the properties of the relations between uniruled and rationally connected varieties (cf. [18, Proposition 3.3]).

Proposition 2.9 (cf. [18]). Suppose $X$ is a variety over a field $k$.

(1) If $X$ is rationally connected, then $X$ is uniruled.

(2) Let $X$ and $X^{\prime}$ be two proper varieties, birational to each other. Then $X$ is rationally connected if and only if so is $X^{\prime}$.

Throughout the proof of Theorem 1.1, we also need several topological facts for rationally connected varieties. Recall that a path-connected topological space $X$ is simply connected if and only if its fundamental group $\pi_{1}(X)$ is trivial. Besides, algebraic 
fundamental group $\pi_{1}^{\text {alg }}(X)$ (cf. [29, Definition 3.5.43]) is a profinite completion of the topological fundamental group $\pi_{1}(X)$ (cf. [29, Theorem 3.5.41]).

Suppose $X$ is a connected variety over a separably closed field. Then comparing with simply connected (with respect to complex topology) varieties, the variety $X$ is said to be algebraically simply connected if it has no nontrivial connected finite étale cover, which is equivalent to $\pi_{1}^{\text {alg }}(X)$ being trivial (cf. [29, Definition 3.5.45]).

Moreover, from Universal Coefficient Theorem for Cohomology (cf. [11, Theorem 3.2]) and Hodge theory, we have the following result.

Lemma 2.10. Suppose $X$ is a smooth variety over $\mathbb{C}$ with trivial fundamental group. Then the irregularity $q(X):=h^{1}\left(X, \mathcal{O}_{X}\right)=\operatorname{dim} \mathrm{H}^{1}\left(X, \mathcal{O}_{X}\right)=0$.

We refer to [31, Theorem 1.1] for the following very useful lemma.

Lemma 2.11 (cf. [31]). Let $X$ be a normal variety and $f: Y \rightarrow X$ a resolution of singularities. Then the induced homomorphism $f_{*}: \pi_{1}(Y) \rightarrow \pi_{1}(X)$ is an isomorphism if the pair $(X, \Delta)$ is klt for some $\Delta$.

In addition, a well-known result (cf. [5, Theorem 3.5] and [16]) gives us when the smooth varieties will be simply connected with respect to complex topology.

Theorem 2.12 (cf. [5] and [16]). A smooth, proper and rationally connected variety is simply connected with respect to complex topology.

2.4. Properties for polarized cases. At the end of this preliminary, we consider a polarized endomorphism $f$ on a normal variety $X$. First, we recall the following result which was proved in [32, Proposition 2.1].

Lemma 2.13 (cf. [32]). Let $X$ be a normal variety, $f: X \rightarrow X$ a surjective endomorphism of $\operatorname{deg}(f)>1$ and $D$ a nonzero reduced divisor with $f^{-1}(D)=D$. Assume:

(1) $X$ is log canonical around $D$;

(2) $D$ is $\mathbb{Q}$-Cartier; and

(3) $f$ is ramified around $D$.

Then the pair $(X, D)$ is log canonical around $D$. In particular, the reduced divisor $D$ is normal crossing outside the union of $\operatorname{Sing} X$ and a codimension three subset of $X$.

For a linear map $\phi: V \rightarrow V$ of a finite dimensional real normed vector space $V$, denote by $\|\phi\|$ the norm of $\phi$. The following proposition gives us a criterion for $\phi$ to be diagonalizable (cf. [23, Definition 2.6, Proposition 2.9] and [6, Proposition 3.1]).

Proposition 2.14 (cf. [6]). Let $\phi: V \rightarrow V$ be an invertible linear map of a positive dimensional real normed vector space $V$. Assume $\phi(C)=C$ for a convex cone $C \subseteq V$ 
such that $C$ spans $V$ and its closure $\bar{C}$ contains no line. Let $q$ be a positive number. Then the conditions (i) and (ii) below are equivalent.

(i) $\phi(u)=q u$ for some $u \in C^{o}$ (the interior part of $C$ ).

(ii) There exists a constant $N>0$, such that $\frac{\left\|\phi^{i}\right\|}{q^{i}}<N$ for all $i \in \mathbb{Z}$. Assume further the equivalent conditions (i) and (ii). Then the following are true.

(1) $\phi$ is a diagonalizable linear map with all eigenvalues of modulus $q$.

(2) Suppose $q>1$. Then for any $v \in V$ such that $\phi(v)-v \in C$, we have $v \in C$.

Corollary 2.15. Suppose $f: X \rightarrow X$ is a polarized endomorphism on a normal projective variety $X$ such that $f^{*} H \equiv q H$ for a positive number $q$ and an ample divisor $H$ on $X$. Then the linear operation $\left.f^{*}\right|_{N_{n-1}(X)}$ is diagonalizable with all eigenvalues of modulus $q$. In particular, if $D$ is an effective reduced Weil divisor such that $f^{-1} D=D$, then with $f$ replaced by its power, $f^{*} D_{i}=q D_{i}$ for each irreducible component $D_{i}$ of $D$ (and then $\left.f^{*} D=q D\right)$.

Proof. We may regard $\mathrm{N}^{1}(X)$ as a subspace of $\mathrm{N}_{n-1}(X)$ (cf. [33, Lemma 3.2]) and consider the invertible linear map $\phi=\left.f^{*}\right|_{\mathrm{N}_{n-1}(X)}$. Let $V:=\mathrm{N}_{n-1}(X)$ and $C:=\mathrm{PE}(X) \subseteq V$ as we defined in Subsection 2.1. Since $H$ is ample, the volume $\operatorname{vol}(H)>0$ and thus $H$ lies in the interior part of $\mathrm{PE}(X)$ if we regard $H$ as a big Weil $\mathbb{R}$-divisor (cf. [8, Theorem 3.5 (ii), (iii)] and [23, Definition 2.4]). In addition, $\operatorname{PE}(X)$ spans the whole $\mathrm{N}_{n-1}(X)$ and then by Proposition $2.14, \phi$ is a diagonalizable linear map with all eigenvalues of modulus $q$.

Since $D$ is $f^{-1}$-invariant, after replacing $f$ by its power, we may assume $f^{-1} D_{i}=D_{i}$ for each irreducible component $D_{i}$ of $D$. Therefore, we can see from the above discussion that $f^{*} D_{i}=\phi\left(D_{i}\right)=q D_{i}$ for each component $D_{i}$.

Remark 2.16. Indeed, the second part of Corollary 2.15 also follows easily from the projection formula (cf. [9, Proposition 2.3]). Besides, the above corollary will fail if we remove the condition that $f$ is polarized. For example (provided by De-Qi Zhang), consider the product $X=\mathbb{P}^{1} \times \mathbb{P}^{1}$ and $f=f_{1} \times f_{2}$, where $f_{i}$ is the power map of $\mathbb{P}^{1}$, mapping $[x, y]$ to $\left[x^{q_{i}}, y^{q_{i}}\right]$ with $q_{1} \neq q_{2}$. Let $D_{1}=\left\{a_{1}\right\} \times \mathbb{P}^{1}, D_{2}=\mathbb{P}^{1} \times\left\{a_{2}\right\}$ and $D=D_{1}+D_{2}$, where $a_{i}$ is one of two $f_{i}^{-1}$-invariant (coordinate) points. Then $D$ is an ample Cartier divisor. However, by projection formula, $f^{*} D_{i}=q_{i} D_{i}$. Moreover, in this case, we can get four $f^{-1}$-invariant prime divisors.

The following proposition extends [32, Proposition 2.12] to the case when $V_{j}$ are reduced divisors. We first state this generalized result and then do some preparations for its proof.

Proposition 2.17. Let $X$ be a normal projective variety of dimension $n \geq 2, V_{j}(1 \leq j \leq$ s) reduced divisors (may have more than one components), and $f: X \rightarrow X$ a polarized endomorphism with $\operatorname{deg}(f)=q^{n}(q>1$ an integer $)$ such that $K_{X}$ is $\mathbb{Q}$-Cartier and 
(1) $X$ has only log canonical singularities around $\bigcup V_{j}$;

(2) every $V_{j}$ is $\mathbb{Q}$-Cartier and ample;

(3) $f^{-1}\left(V_{j}\right)=V_{j}$ for all $j$; and

(4) $V_{i}$ and $V_{j}(i \neq j)$ have no common irreducible components.

Then $s \leq n+1$; and $s=n+1$ only if: $f$ is étale outside $\left(\bigcup V_{j}\right) \cup f^{-1}(\operatorname{Sing} X)$ and $K_{X}+\sum_{j=1}^{n+1} V_{j} \equiv 0$.

Remark 2.18. With the same assumption in Proposition 2.17, the remaining necessary condition for $s=n+1$ in [32, Proposition 2.12] still holds: each $V_{j}$ is irreducible and $\bigcap_{i=1}^{t} V_{b_{i}} \subseteq X$ is a normal irreducible subvariety for every subset $\left\{b_{1}, \cdots, b_{t}\right\} \subseteq\{1, \cdots, n+$ $1\}$ with $1 \leq t \leq n-2$ (cf. [32, Claim 2.11], Proof of Proposition 2.17 and Remark 2.21).

Before proving Proposition 2.17, we first prove the following lemmas. Lemma 2.19 follows immediately from Corollary 2.15.

Lemma 2.19. Suppose $f: X \rightarrow X$ is a polarized endomorphism on a normal projective variety $X$ of dimension $n$ and $\operatorname{deg} f=q^{n}>1$. Then any Weil divisor $M$ (not necessarily effective) on $X$ such that $f^{*} M \equiv_{w} M$, is weakly numerically trivial. In particular, suppose further that $M$ is $\mathbb{Q}$-Cartier. Then $M \equiv 0$.

Proof. Since $f$ is polarized by an ample divisor $H$ on $X$, the linear operation $\left.f^{*}\right|_{\mathrm{N}_{n-1}(X)}$ is diagonalizable with all eigenvalues of modulus $q>1$ (cf. Corollary 2.15). Suppose $M \not \equiv_{w} 0$. Then 1 is an eigenvalue of the linear operation $\left.f^{*}\right|_{\mathrm{N}_{n-1}(X)}$, a contradiction. Therefore, $M \equiv_{w} 0$. Further, if $M$ is $\mathbb{Q}$-Cartier, then $M \equiv 0$ (cf. [33, Lemma 3.2]).

We follow the idea of [32, Lemma 2.7] to prove the following result.

Lemma 2.20. Suppose $f: X \rightarrow X$ is a finite surjective endomorphism on a normal projective variety $X$ of dimension $n \geq 1$. Suppose further that $M$ is a pseudo-effective Weil divisor and $E$ is an effective $\mathbb{Q}$-divisor such that the following weakly numerical equivalence $(*)$ holds:

$$
\text { (*) } M \equiv_{w} f^{*} M+E \text {. }
$$

Then the effective $\mathbb{Q}$-divisor $E=0$.

Proof. Suppose $E>0$. Multiplying $(*)$ by a positive integer, we may assume $E$ is integral. Substituting the above expression of $M$ to the right-hand side $(k-1)$-times, we get

$$
M \equiv_{w}\left(f^{k}\right)^{*} M+\sum_{i=0}^{k-1}\left(f^{i}\right)^{*} E .
$$


Taking a fixed ample Cartier divisor $H$ on $X$ and then using Nakai-Moishezon criterion (cf. [17, Theorem 1.37]), we have the following

$$
\left(M \cdot H^{n-1}\right) \geq \sum_{i=0}^{k-1}\left(\left(f^{i}\right)^{*} E \cdot H^{n-1}\right) .
$$

Since $E$ is integral, the right-hand side tends to infinity if we let $k \rightarrow \infty$, a contradiction. Hence, $E=0$.

Now, we begin with the proof of Proposition 2.17. We follow the steps and use the similar method given in [32, the proof of Proposition 2.12]. Besides, readers may refer to [32, Lemma 2.8] for a further proof of Remark 2.18.

Proof. Suppose $s \geq n+1$. If $n=2$, then we may go to the end product $X_{n-2}$ with $n=2$. Therefore, we may further assume that initially $n \geq 3$ and $f^{*} H \sim q H$ for an ample divisor $H$ on $X$. Since the number of $V_{j}$ and the irreducible components of $V_{j}$ are finite, with $f$ replaced by its power, we may assume for each irreducible component $B_{i j}$ of $V_{j}, f^{-1}\left(B_{i j}\right)=B_{i j}$. By Corollary 2.15, $f^{*} B_{i j}=q B_{i j}$. Note that Proposition 2.17 still holds with $f$ replaced by its power since $f$ is étale away from $\cup V_{j}$ if so is its power.

We reduce the dimension of $X$ by continuously taking normalization of divisors. Then we prove Proposition 2.17 by an early result for the surface case. Let $D_{0}=\sum V_{j}$ and consider the log ramification divisor formula for the pair $\left(X, D_{0}\right)$ (cf. [12, Theorem 11.5]):

$$
K_{X}+D_{0}=f^{*}\left(K_{X}+D_{0}\right)+\Delta_{f}
$$

where $\Delta_{f}$ is an effective (integral) divisor, having no common components with $D_{0}$.

Suppose further $\Delta_{f}>0$. Since $V_{1}$ is ample, $\left(V_{1}^{n-1} \cdot \Delta_{f}\right)>0$. Fix a component $B_{0}$ of $V_{1}$ intersecting $\Delta_{f}$ and take the normalization of $B_{0}$ followed by the inclusion map:

$$
\sigma_{1}: X_{1}=\widetilde{B_{0}} \rightarrow B_{0} \hookrightarrow X_{0}=X .
$$

Then one can get a commutative diagram (by the universal property of normalization):

$$
f_{1}^{*}\left(\sigma_{1}^{*} H\right)=\sigma_{1}^{*}\left(f^{*} H\right) \sim q \sigma_{1}^{*} H
$$

which means that this lifting $f_{1}$ is polarized by $\sigma_{1}^{*} H$ and $\operatorname{deg} f_{1}=q^{n-1}$. Pulling back Equation (1) along the map $\sigma_{1}$, we have the following:

$$
K_{X_{1}}+D_{1}:=\sigma_{1}^{*}\left(K_{X}+D_{0}\right)=\sigma_{1}^{*}\left(f^{*}\left(K_{X}+D_{0}\right)+\Delta_{f}\right)=f_{1}^{*}\left(K_{X_{1}}+D_{1}\right)+\sigma_{1}^{*} \Delta_{f} .
$$

Here, $D_{1}=\operatorname{Diff}\left(D_{0}\right)$ contains the reduced Weil divisor $\sum_{j \geq 2} \operatorname{Supp} \sigma_{1}^{*} V_{j}$ (cf. [30, Corollary 3.11], [15, Corollary 16.7] and Lemma 2.13). Besides, $\left(X_{1}, D_{1}\right)$ is log canonical by Proposition 2.1. Note that $\left.V_{j}\right|_{B_{0}}(j>1)$ is ample on $B_{0}$ and the normalization is a finite surjective morphism. Hence, each $\sigma_{1}^{*} V_{j}(j>1)$ is nonzero and still ample on $X_{1}$ (cf. [17, 
Theorem 1.37]). Moreover, $\operatorname{Supp} \sigma_{1}^{*} V_{j}(j>1)$ is connected since $\operatorname{dim} X_{1}=n-1 \geq 2$ (cf. [10, Corollary 7.9]).

By the choice of $B_{0},\left.\Delta_{f}\right|_{B_{0}}$ is a nonzero effective $\mathbb{Q}$-divisor on $B_{0}$. Since the normalization is finite, $\sigma_{1}^{*} \Delta_{f}$ is a nonzero effective $\mathbb{Q}$-divisor on $X_{1}$. Repeatedly, we fix an (integral) irreducible component $B_{1}$ of $\operatorname{Supp} \sigma_{1}^{*} V_{2}\left(\leq D_{1}\right)$ intersecting $\sigma_{1}^{*} \Delta_{f}$. This is possible since $\sigma_{1}^{*} V_{2}$ is ample on $X_{1}$. With $f$ replaced by its power, we may assume $f_{1}^{-1}\left(B_{1}\right)=B_{1}$. Since $f_{1}$ is polarized, by Corollary 2.15, we have $f_{1}^{*} B_{1}=q B_{1}$. Taking the normalization of $B_{1}$ followed by the inclusion map, we get $\sigma_{2}: X_{2} \rightarrow X_{1}$ with $\operatorname{dim} X_{2}=n-2$. Similarly, $\left.f_{1}\right|_{B_{1}}$ lifts to a polarized endomorphism $f_{2}$ of $X_{2}$ with $\operatorname{deg} f_{2}=q^{n-2}$.

In general, let $\sigma_{i}: X_{i} \rightarrow X_{i-1}(1 \leq i \leq n-2)$ be the normalization of an (integral) irreducible component $B_{i-1}$ of $\operatorname{Supp}\left(\sigma_{1} \circ \cdots \circ \sigma_{i-1}\right)^{*} V_{i}\left(\leq D_{i-1}\right)$ intersecting $\left(\sigma_{1} \circ \cdots \circ\right.$ $\left.\sigma_{i-1}\right)^{*} \Delta_{f}$ followed by an inclusion map. Then we get $f_{i}: X_{i} \rightarrow X_{i}$ polarized by the pullback $\left(\sigma_{1} \circ \cdots \circ \sigma_{i}\right)^{*} H, \operatorname{dim} X_{i}=n-i$ and $\operatorname{deg} f_{i}=q^{n-i}$ (cf. [28, Lemma 2.1]). Let $\sigma$ be the composition,

$$
\sigma:=\sigma_{1} \circ \cdots \sigma_{n-2}: X_{n-2} \longrightarrow X_{0}=X
$$

Now, $S:=X_{n-2}$ is a normal surface with following ample reduced divisors on $S$ :

$$
C_{i}:=\operatorname{Supp} \sigma^{*} V_{i}(n-1 \leq i \leq s)
$$

After replacing $f$ by its power, we may assume $f_{n-2}^{*} C_{i j}=q C_{i j}$ for each irreducible component $C_{i j}$ of $C_{i}(i \geq n-1)$. On the one hand, using the log ramification divisor formula for the pair $\left(S, C:=\sum_{i \geq n-1} C_{i}\right)$ (cf. [12, Theorem 11.5]), we have

$$
K_{S}+C=f_{n-2}^{*}\left(K_{S}+C\right)+\Delta .
$$

Here, $\Delta$ is an effective (integral) Weil divisor, sharing no common components with $C$. On the other hand, pulling back Equation (1) along the map $\sigma$, we get

$$
K_{S}+D_{n-2}:=\sigma^{*}\left(K_{X}+D_{0}\right)=f_{n-2}^{*}\left(K_{S}+D_{n-2}\right)+\sigma^{*} \Delta_{f}
$$

Comparing Equation (4) with (5), we get $C \leq D_{n-2}$ (cf. [30, Corollary 3.11] and [15, Corollary 16.7]), and thus $M:=D_{n-2}-C \geq 0$. By assumption, the number of $C_{i}$, $r \geq s-(n-2) \geq 3$.

We will show that $s=n+1$. Indeed, note that each $C_{i}(n-1 \leq i \leq s)$ is connected and ample, so $\left(C_{i} \cdot C_{j}\right)>0$ for $n-1 \leq i, j \leq s$. Let $D=\sum_{j=n-1}^{n+1} C_{j} \leq C$. Then the dual graph of $D$ contains a loop. By [32, Lemma 2.8], $K_{S}+D \sim 0$. Back to Equation (5), we have the following:

$$
M+C-D \equiv_{w} f_{n-2}^{*}(M+C-D)+\sigma^{*} \Delta_{f}
$$


Since $\sigma^{*} \Delta_{f}$ is an effective $\mathbb{Q}$-divisor, by Lemmas 2.19 and $2.20, M+C-D \equiv_{w} 0$ and $\sigma^{*} \Delta_{f}=0$. Thus, $M=C-D=0$ by the effectivity of $M$ and $C-D$. As a result, we have $D_{n-2}=C=D$, which in turn implies that $s=n+1$.

Recall our initial assumption that $\Delta_{f} \neq 0$. Then by our choice of each $B_{i}$ and $\sigma, \sigma^{*} \Delta_{f}$ is a nonzero effective $\mathbb{Q}$-divisor on $S$. However, by Equation (6) and Lemma 2.20, we have already got $\sigma^{*} \Delta_{f}=0$, a contradiction. Therefore, $\Delta_{f}=0$ and the ramification divisor of $f, R_{f}=(q-1) D_{0}$. By the purity of branch loci, $f$ is étale outside $\left(\bigcup V_{j}\right) \cup f^{-1}(\operatorname{Sing} X)$. Further, since $\Delta_{f}=0$, we get $K_{X}+D_{0} \equiv 0$ (cf. Equation (1) and Lemma 2.19), which completes the proof of Proposition 2.17.

Remark 2.21. For Proposition 2.17, when the equality holds, i.e. $s=n+1$, we claim that $D_{1}$ (and then each $D_{i}$ ) is reduced. With the same symbols given above, we follow the ideas and steps of [32, the proof of Theorem 1.1] to prove it as follows.

Proof. Note that $\Delta_{f}=0$. Suppose $\Gamma_{1}=m F_{1} \leq D_{1}$ is a non-reduced fractional component with $m<1$. Since $\sigma_{1}^{*} V_{2}$ is ample on $X_{1}$, the intersection number $\left(\Gamma_{1} \cdot\left(\sigma_{1}^{*} V_{2}\right)^{\operatorname{dim} X_{1}-1}\right)>0$. Therefore, there exists an (integral) irreducible component of $\operatorname{Supp} \sigma_{1}^{*} V_{2}\left(\leq D_{1}\right)$, intersecting $F_{1}$. Let this component be our new $B_{1}$ as in the proof of Proposition 2.17. Then taking the normalization of $B_{1}$ followed by the inclusion map, we get

$$
K_{X_{2}}+D_{2}=f_{2}^{*}\left(K_{X_{2}}+D_{2}\right) .
$$

Then we get the nonzero pullback $\Gamma_{2}^{\prime}:=\sigma_{2}^{*} \Gamma_{1} \leq D_{2}$ by the choice of $B_{1}$. Fix an irreducible component $\Gamma_{2}$ of $\Gamma_{2}^{\prime}$, which has no common components with $\operatorname{Supp} \sigma_{1}^{*} V_{j}(j \geq n-1)$.

In general, suppose we have fixed a component $\Gamma_{k}$ of the pullback of $\Gamma_{k-1}$. Consider the pullback of $V_{k+1}$, which is ample on $X_{k}$. Choosing the (integral) irreducible component $B_{k}$ which intersects $\Gamma_{k}$, we take the normalization of $B_{k}$ followed by the inclusion.

Finally, we get a component $\Gamma_{n-2} \leq D_{n-2}$ on $S$. Since we have proved that $\Delta_{f}=0$ for the case when $s=n+1$ and it is independent of our choice of $B_{i}$ and $\sigma$, we get

$$
K_{S}+D_{n-2}=f_{n-2}^{*}\left(K_{S}+D_{n-2}\right) .
$$

Note that $K_{S}+D_{n-2}$ is the pullback of $K_{X}+D_{0}$ under our new $\sigma$. Similarly, comparing the above equation with Equation (4) and (5), we have $M=D_{n-2}-C \geq 0$ and $f_{n-2}^{*} M=$ $M$. Therefore, $M$ is numerically trivial (cf. Lemma 2.19), which in turn gives $M=0$. Therefore, $C=D_{n-2}$ and such $\Gamma_{n-2}$ cannot exist since $C$ is the support of the pullback of $\sum_{j=n-1}^{n+1} V_{j}$ and thus there is no more space for $\Gamma_{n-2}$. As a result, our assumption is absurd.

The following lemma is known to Iitaka, Sommense, Fujimota and Nakayama (cf. [27, Lemma 3.7.1]), we rewrite it for the convenience of readers. 
Lemma 2.22 (cf. [27]). Let $X$ be a normal projective variety of dimension $n$ and $f$ : $X \rightarrow X$ an endomorphism with $\operatorname{deg}(f) \geq 2$. Suppose the canonical divisor $K_{X}$ is a pseudo-effective $\mathbb{Q}$-Cartier divisor. Then $f$ is étale in codimension one.

\section{Proof of Theorem 1.1}

In this section, we begin with our proof of Theorem 1.1. We follow the steps in [32, The proof of Theorem 1.3] for the case of polarized endomorphisms. The main idea is to run MMP and reduce the dimension of $X$ gradually. Then we use the induction. In the beginning, we introduce a key proposition (cf. [32, The proof of Lemma 2.8]).

Proposition 3.1 (cf. [32]). Suppose $X$ is a rationally connected variety with at worst klt singularities. Then the Picard group $\operatorname{Pic}(X)$ is torsion free and $\operatorname{Pic}(X) \cong \mathrm{NS}(X)$.

To prove Proposition 3.1, we need the following well-known fact.

Lemma 3.2. Suppose $X$ is a normal projective variety. Then the set of $\mathbb{C}$-points $X(\mathbb{C})$ is path-connected with respect to complex topology.

Now, we prove Proposition 3.1.

Proof. Take any resolution $g: S \rightarrow X$. Then since $g$ is birational, $S$ is smooth and rationally connected by Proposition 2.9. Also, Theorem 2.12 tells us that $S$ has a trivial fundamental group. By Lemma 2.10, the irregularity of $S, q(S)=0$. Now, since $X$ is klt, we have $q(X)=q(S)=0$ (cf. [17, Theorem 5.22 and Definition 5.8]), which implies that $\operatorname{Pic}^{\circ}(X)=0$. Moreover, by Lemma $2.11, \pi_{1}(X) \cong \pi_{1}(S)=\{1\}$.

Now, since $\operatorname{Pic}^{\circ}(X)=0, \operatorname{Pic}(X) \cong \operatorname{NS}(X)$, which is finitely generated. Suppose there exists some invertible sheaf $\mathcal{L}=\mathcal{O}(D)$, where $D$ is Cartier such that $n D \sim 0$ for some (minimal) integer $n \geq 1$. Then there exists an unramified cyclic cover $p: X_{n, D} \rightarrow X$ of degree $n$, which is a finite étale morphism (cf. [17, Definition 2.49]). Since $X$ is normal and projective, $X(\mathbb{C})$ is path-connected by Lemma 3.2. Then $\pi_{1}(X)=\{1\}$ implies that $X$ is simply connected with respect to complex topology and thus algebraically simply connected, which means there does not exist any nontrivial connected finite étale cover. Therefore, $n=1$ and $\mathcal{O}(D)$ is trivial. In conclusion, the Picard group of any rationally connected variety with only klt singularities over $\mathbb{C}$ is torsion free. This completes the proof of Proposition 3.1.

Now, we use the induction on $\operatorname{dim} X$ to prove Theorem 1.1.

3.1. The Case $\operatorname{dim} X=1$.

Lemma 3.3. Theorem 1.1 holds for the case when $\operatorname{dim} X=1$. 
In this case, $X$ being normal is equivalent to $X$ being smooth. We give the proof of Lemma 3.3 as follows.

Proof. It's well known that a curve with the genus $g(X)>1$ does not admit any nonisomorphic surjective endomorphisms. Since $f: X \rightarrow X$ is int-amplified, $\operatorname{deg} f>1$ (cf. [22, Lemma 3,7]). Hence, there are two cases.

Case (a). The genus of $X, g(X)=1$, i.e. $X$ is an elliptic curve. In this case, Theorem 1.1 (1) naturally holds, since $c=0$ by Hurwitz's Theorem. For Theorem 1.1 (2), since $\mathrm{NS}(X) \cong \mathbb{Z}$ for the elliptic curve $X$ (cf. [25, pp.165 Corollary 2]), $\left.f^{*}\right|_{\mathrm{NS}_{\mathbb{Q}}(X)}$ is diagonalizable over $\mathbb{Q}$. Further, Theorem 1.1 (3) and (4) cannot happen in this case.

Case (b). The genus of $X, g(X)=0$. Then $X \cong \mathbb{P}^{1}$. By Hurwitz's Theorem,

$$
K_{X}+\sum V_{i}=f^{*}\left(K_{X}+\sum V_{i}\right)+R_{f}^{\prime}
$$

with $R_{f}^{\prime}$ effective. Taking the degree of both sides, we have

$$
-2+c=(\operatorname{deg} f)(-2+c)+\operatorname{deg} R_{f}^{\prime} .
$$

Therefore, $c \leq 2$. In this case, Theorem $1.1(2),(3),(4)$ obviously hold since $\operatorname{Pic}\left(\mathbb{P}^{1}\right) \cong \mathbb{Z}$. For Theorem 1.1 (1), computing the discrepancy (cf. [17, Corollary 2.31]), we see that $\left(X, \sum V_{i}\right)$ is $\log$ canonical. Moreover, $X \cong \mathbb{P}^{1}$ is rational, and then it is uniruled. Thus, we have completed the proof of Theorem 1.1 for the case when $\operatorname{dim} X=1$.

3.2. The Case $\operatorname{dim} X \geq 2$. Suppose Theorem 1.1 holds for those $X^{\prime}$ with $\operatorname{dim} X^{\prime} \leq n-1$. From now on, we consider the case when $\operatorname{dim} X=n \geq 2$. We assume that $V_{j}(1 \leq j \leq c)$ are all the prime divisors in $\mathrm{TI}_{f}(X)$, which are contained in the ramification divisors of $f$ (cf. Lemma 2.6). According to the hypotheses in our theorem, we may assume $c \geq \rho(X)+n-2 \geq 1$ (and hence $K_{X}$ is not pseudo-effective by Lemma 2.22).

During the proof of Theorem 1.1, for (1) and (4), we only need one Fano contraction when running MMP while for (2) and (3), we need the end product $Y$ of MMP in Theorem 2.3. The detailed proof is as follows.

3.2.1. Minimal Model Program for $X$. By Theorem 2.3, if $K_{X}$ is pseudo-effective, then $X$ is $Q$-abelian. From now on, we assume that $K_{X}$ is not pseudo-effective. Then the minimal model program of $X$ will end with a Fano contraction $X_{l} \rightarrow Y$ (cf. [Ibid.]). For each $u_{i}: X_{i} \rightarrow X_{i+1}$, it is one of the following types: a divisorial contraction, a flip or a Fano contraction of a $K_{X_{i}}$-negative extremal ray. Furthermore, there exists a positive integer $t$, such that $g_{0}=f^{t}$ descends to an int-amplified endomorphism $g_{i}$ of $X_{i}$ for each $1 \leq i \leq l+1$. 
By the log ramification divisor formula for the pair $\left(X, \sum V_{j}\right)$ (cf. [12, Theorem 11.5]),

$$
K_{X}+\sum V_{j}=f^{*}\left(K_{X}+\sum V_{j}\right)+\Delta_{f},
$$

with $\Delta_{f}$ effective, having no common components with $\bigcup \operatorname{Supp} V_{j}$. Note that each $V_{j}$ is $f^{-1}$-invariant and thus $g_{0}^{-1}$-invariant, i.e. $\mathrm{TI}_{f}(X) \subseteq \mathrm{TI}_{g_{0}}(X)$.

For each $u_{i}$, the Picard number will decrease by one if $u_{i}$ is a divisorial contraction or a Fano contraction while the Picard number will be the same if $u_{i}$ is a flip (cf. [17, Proposition 3.36, 3.37]). This point provides the possibility for our induction.

We focus on a specific case. When running MMP, once there appears a Fano contraction, one stops it immediately. In other words, we consider the composite morphisms:

$$
X=X_{0}-->X_{1}-->\cdots-->X_{i}-->\cdots-->X_{r}-->X_{r+1}=Y_{0}
$$

where each $u_{i}: X_{i} \rightarrow X_{i+1}(0 \leq i \leq r-1)$ is either a divisorial contraction or a flip and $u_{r}: X_{r} \rightarrow Y_{0}$ is a Fano contraction. Then each $u_{i}(0 \leq i \leq r-1)$ is a birational map and the dimensions of $X_{i}(0 \leq i \leq r)$ are the same.

Let $V(1)_{j} \subseteq X_{1}$ be the strict transform of $V_{j}$ under the birational map $u_{0}$ if $V_{j}$ is not exceptional over $X_{1}$. Then $V(1)_{j}$ is still a prime divisor. Since $g_{0}$ descends to an int-amplified endomorphism $g_{1}$ on $X_{1}$ by Theorem 2.3, $V(1)_{j} \in \mathrm{TI}_{g_{1}}\left(X_{1}\right)$. Using Lemma 2.6, we get $g_{1}^{*}\left(V(1)_{j}\right)=\alpha_{j} V(1)_{j}$ for some $\alpha_{j}>1$.

In general, for each $0 \leq i \leq r-1$, let $V(i+1)_{j}$ be the strict transform of $V(i)_{j}$ under the birational map $u_{i}$ if $V(i)_{j}$ is not exceptional over $X_{i+1}$. Then $V(i+1)_{j} \in \mathrm{TI}_{g_{i+1}}\left(X_{i+1}\right)$.

We use $s_{i}(0 \leq i \leq r)$ to denote the cardinality of $\mathrm{TI}_{g_{i}}\left(X_{i}\right)$. It is obvious that $s_{0} \geq c$. Also, for each $i$, the number of such $V(i)_{j}$ cannot exceed $s_{i}$. By the log ramification divisor formula for the pair $\left(X_{i}, \sum_{j} V(i)_{j}\right)$ (cf. [12, Theorem 11.5]), we get

$$
K_{X_{i}}+\sum_{j} V(i)_{j}=g_{i}^{*}\left(K_{X_{i}}+\sum_{j} V(i)_{j}\right)+\Delta(i)
$$

Here, $\Delta(i)$ is an integral log ramification divisor having no common components with $\sum V(i)_{j}$.

Lemma 3.4. With the same symbols given above, the equality $s_{1}=s_{0}-\left(\rho\left(X_{0}\right)-\rho\left(X_{1}\right)\right)$ holds (and thus, $s_{i}=s_{0}-\left(\rho\left(X_{0}\right)-\rho\left(X_{i}\right)\right)$ for each $\left.i \leq r\right)$. In particular, if $c \geq$ $\rho\left(X_{0}\right)+\operatorname{dim} X_{0}-k$ for some fixed integer $k$, then $s_{r} \geq \rho\left(X_{r}\right)+\operatorname{dim} X_{r}-k$.

Proof. We use the induction to prove our claim. There are two cases for $u_{0}$ :

Case (1): Suppose $u_{0}$ is a divisorial contraction. Then, since $u_{0}$ is $g_{0}=f^{t}$ equivariant, the exceptional divisor $E$ of $u_{0}$ lies in $\mathrm{TI}_{g_{0}}\left(X_{0}\right)$. Thus, $s_{1}=s_{0}-1$ and also $\rho\left(X_{1}\right)=$ $\rho\left(X_{0}\right)-1$. In this case, we get $s_{1}=s_{0}-\left(\rho\left(X_{0}\right)-\rho\left(X_{1}\right)\right)$. 
Case (2): Suppose $u_{0}$ is a flip. Then $u_{0}$ is an isomorphism in codimension one. Therefore, $u_{0}$ does not contract or create any new divisors. Then $s_{0}=s_{1}$, and $\rho\left(X_{1}\right)=\rho\left(X_{0}\right)$. In this case, $s_{1}=s_{0}-\left(\rho\left(X_{0}\right)-\rho\left(X_{1}\right)\right)$ still holds.

In any case, we get the same equality for $X_{1}$. Repeating the above discussion, we complete the first part of our claim. For the second part, note that $s_{0} \geq c$ and $\operatorname{dim} X_{i}=n$ for each $i \leq r$. Hence, the inequality follows from the above equality for each $i \leq r$.

Lemma 3.5. With the same symbols given above, suppose $\Delta(r)=0$ for $i=r$ in Equation (8). Then the following statements hold.

(1) $K_{X_{r}}+\sum_{j} V(r)_{j} \equiv 0$.

(2) If $\operatorname{Pic}^{\circ}\left(X_{r}\right)=0$ and every exceptional divisor of the composite map: $\delta: X_{0} \rightarrow X_{r}$ is contained in $\mathrm{TI}_{f}\left(X_{0}\right)$, where $\delta=u_{r-1} \circ \cdots \circ u_{0}$, then $\Delta_{f}=0$ in Equation (7) and hence $K_{X}+\sum V_{j} \equiv 0$. Moreover, $f$ is étale outside $\left(\cup V_{j}\right) \cup f^{-1}(\operatorname{Sing} X)$.

We will apply the above result to prove Theorem 1.1 (4). Before proving Lemma 3.5, we refer readers to the following remarks on the assumption of this lemma.

Remark 3.6. A prime divisor $E$ on $X_{0}$ is $\delta$-exceptional if it is exceptional over some $X_{i}$. Suppose $E \subseteq X_{i-1}$ is exceptional over $X_{i}$. Then for the divisorial contraction $u_{i-1}$ : $X_{i-1} \rightarrow X_{i}$, the image $u_{i-1}(E)$ is a codimension $\geq 2$ closed subset of $X_{i}$. So it cannot create new divisors when mapped into $X_{i+1}$ no matter $u_{i}$ is a divisorial contraction or a flip, since divisorial contractions are birational and flips are isomorphic in codimension one. Besides, we define the pullback of $\delta$ (cf. Equation (10)) as the composite of $u_{0}^{*} \circ u_{1}^{*} \circ$ $\cdots \circ u_{r-1}^{*}$. Moreover, $\operatorname{NS}\left(X_{0}\right)$ is generated by the pullback of generators of $\operatorname{NS}\left(X_{r}\right)$ and all the $\delta$-exceptional divisors (cf. [17, Proposition 3.36 and 3.37]).

The composite $\delta: X=X_{0} \rightarrow X_{r}$ is always $g_{0}$-equivariant by the choice of our $g_{0}$, i.e. $g_{0}$ descends to an int-amplified endomorphism $g_{r}$ on $X_{r}$. Hence, every exceptional divisor $E$ of $\delta$ is contained in $\operatorname{TI}_{g_{0}}\left(X_{0}\right)$. However, this is not enough to conclude our result, and the assumption of (2) in Lemma 3.5 is necessary. We will see later that the assumption of (2) holds when the cardinality $c$ of $\mathrm{TI}_{f}\left(X_{0}\right)$ achieves the upper bound $\operatorname{dim} X_{0}+\rho\left(X_{0}\right)$.

Proof. We begin to prove Lemma 3.5. Indeed, Lemma 3.5 (1) follows immediately from Lemma 2.6. Now, we prove (2). Since $\operatorname{Pic}^{\circ}\left(X_{r}\right)=0$, (1) implies that

$$
K_{X_{r}}+\sum V(r)_{j} \sim_{\mathbb{Q}} 0 .
$$

Write down the well-defined $\delta$-pullback formula as follows:

$$
K_{X}+\sum V_{j}=\delta^{*}\left(K_{X_{r}}+\sum V(r)_{j}\right)+E_{2}-E_{1} .
$$


Here, $E_{k}=\sum_{l} E_{k l}(k=1,2)$ are effective $\delta$-exceptional divisors and each $E_{k l}$ lies in $\mathrm{TI}_{f}\left(X_{0}\right)$ by assumption. Hence, we may assume $f^{*} E_{k}=\sum_{l} a_{k l} E_{k l}$ with $a_{k l}>1$. Recall the log ramification divisor formula for $\left(X, \sum V_{j}\right)$ (cf. Equation (7)):

$$
K_{X}+\sum V_{j}=f^{*}\left(K_{X}+\sum V_{j}\right)+\Delta_{f}
$$

with $\Delta_{f}$ effective, having no common components with $\bigcup V_{i}$. In summary, $\operatorname{Supp} E_{1}$ and $\operatorname{Supp}\left(E_{2}+\Delta_{f}\right)$ have no common components and $\operatorname{Supp}\left(E_{1}+E_{2}\right) \subseteq \bigcup V_{j}$, since $V_{j}(1 \leq j \leq c)$ are all the prime divisors in $\mathrm{TI}_{f}(X)$. By Equation (7), (9) and (10),

$$
E_{2}-E_{1}-\Delta_{f} \sim_{\mathbb{Q}} f^{*}\left(E_{2}-E_{1}\right)=\sum a_{2 l} E_{2 l}-\sum a_{1 l} E_{1 l} .
$$

Therefore, we get the following equation with both sides effective:

$$
\Delta_{f}+\sum\left(a_{2 l}-1\right) E_{2 l} \sim_{\mathbb{Q}} \sum\left(a_{1 l}-1\right) E_{1 l} .
$$

We claim that $E_{1}=E_{2}=\Delta_{f}=0$. Suppose the contrary that the claim does not hold. Let $F_{k}:=\sum_{l}\left(a_{k l}-1\right) E_{k l} \geq 0$ for $k=1,2$. On the one hand, since Supp $F_{1}$ and $\operatorname{Supp}\left(\Delta_{f}+F_{2}\right)$ have no common components, Equation (12) gives us that $\kappa\left(X, F_{1}\right) \geq 1$. On the other hand, we take the graph of the birational map $\delta: X-->X_{r}$ :

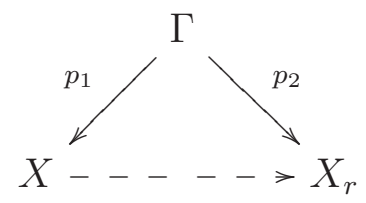

Here, the projections $p_{1}$ and $p_{2}$ are birational morphisms. As we mentioned above, $F_{k}$ is $\delta$-exceptional for $k=1,2 ; \delta$ is a composite of flips or birational morphisms and thus $\delta$ does not extract divisors. Therefore, $p_{2 *} p_{1}^{*} F_{1}=0$. As a result, we have the following:

$$
\mathrm{H}^{0}\left(X, \mathcal{O}_{X}\left(m F_{1}\right)\right)=\mathrm{H}^{0}\left(\Gamma, p_{1}^{*} \mathcal{O}_{X}\left(m F_{1}\right)\right)=\mathrm{H}^{0}\left(X_{r}, p_{2 *} p_{1}^{*} \mathcal{O}_{X}\left(m F_{1}\right)\right)=\mathrm{H}^{0}\left(X_{r}, \mathcal{O}_{X_{r}}\right)=k
$$

for any $m>0$. The first equality is due to projection formula. By definition, the Iitaka dimension $\kappa\left(X, F_{1}\right)=0$, a contradiction. Therefore, our claim holds.

Since $\Delta_{f}=0$ in Equation (7), applying Lemma 2.6 to Equation (7), $K_{X}+\sum V_{j} \equiv 0$. Moreover, $\Delta_{f}=0$ implies that the ramification divisor of $f$ consists only of these $V_{j}$ 's. By the purity of branch loci, $f$ is étale outside $\left(\cup V_{j}\right) \cup f^{-1}(\operatorname{Sing} X)$, which completes the proof of Lemma 3.5.

3.2.2. The Case of $\operatorname{dim} Y_{0}=0$. In this case, $\rho\left(X_{r}\right)=1$ and $-K_{X_{r}}$ is ample. Then $X_{r}$ is $\log Q$-Fano and thus rationally connected (cf. [34]). Since $X$ and $X_{r}$ are birational, $X$ is also rationally connected (and hence uniruled) by Proposition 2.9. Besides, $X$ is simply connected with respect to complex topology (cf. Theorem 2.12 and Lemma 2.11) and $X_{r}$ 
also has only klt singularities (cf. [17, Corollary 3.42, 3.43]). Then, by Proposition 3.1, $\operatorname{Pic}^{\circ}\left(X_{r}\right)=\operatorname{Pic}^{\circ}\left(X_{0}\right)=0, \operatorname{Pic}\left(X_{0}\right) \cong \operatorname{NS}\left(X_{0}\right)$ and $\operatorname{Pic}\left(X_{r}\right) \cong \operatorname{NS}\left(X_{r}\right)$.

We fix an ample divisor $H_{X_{r}}$ on $X_{r}$. Since $\rho\left(X_{r}\right)=1$, we get $g_{r}^{*} H_{X_{r}} \equiv a H_{X_{r}}$ and also $a>1$ (cf. Lemma 2.6). Hence $g_{r}$ is polarized by [23, Lemma 2.3]. Further, since $\operatorname{Pic}^{\circ}\left(X_{r}\right)=0$, with $H_{X_{r}}$ replaced by its power, we may assume $g_{r}^{*} H_{X_{r}} \sim a H_{X_{r}}$. By Proposition 2.9 and Remark 3.6, $\operatorname{Pic}\left(X_{0}\right) \otimes_{\mathbb{Z}} \mathbb{Q} \cong \mathrm{NS}_{\mathbb{Q}}\left(X_{0}\right)$ as a $\mathbb{Q}$-vector space, is spanned by $\delta$-exceptional divisors and $\delta^{*} H_{X_{r}}$ over $\mathbb{Q}$. On the one hand, $g_{0}^{*}\left(\delta^{*}\left(H_{X_{r}}\right)\right)=\delta^{*}\left(g_{r}^{*}\left(H_{X_{r}}\right)\right) \sim$ $a \delta^{*}\left(H_{X_{r}}\right)$ for some $a>1$. On the other hand, for each $\delta$-exceptional prime divisor $E$, it is contained in $\mathrm{TI}_{g_{0}}\left(X_{0}\right)$ by the choice of $g_{0}$ and $g_{0}^{*} E=\beta E$ for some $\beta>1$. Therefore, $\left.g_{0}^{*}\right|_{\mathrm{Pic} X}$ is diagnolizable over $\mathbb{Q}$. Since $g_{0}$ is a positive power of $f$, this completes the proof of Theorem $1.1(2)$ and (3) for the case when $\operatorname{dim} Y_{0}=0$.

Suppose $c \geq \rho(X)+n$ from now on. Then $s_{r} \geq n+\rho\left(X_{r}\right)=n+1$ (cf. Lemma 3.4). Since $g_{r}$ is polarized and each $V(r)_{j}$ is ample, by Proposition 2.17, we get

$$
s_{r}=n+1, K_{X_{r}}+\sum_{j=1}^{n+1} V(r)_{j} \equiv 0 .
$$

Therefore, by [1, CH. XIII, Theorem 4.6] (and $\operatorname{Pic}^{\circ}\left(X_{r}\right)=0$ ), we also get Equation (9). Since $s_{r}=n+1=n+\rho\left(X_{r}\right)$, by Lemma 3.4, $s_{i}=n+\rho\left(X_{i}\right)$ for each $0 \leq i \leq r$. The equality for the case when $i=0$ in turn forces $s_{0}=c$. Thus, $\operatorname{TI}_{f}\left(X_{0}\right)=\operatorname{TI}_{g_{0}}\left(X_{0}\right)$, i.e. all of these $g_{0}^{-1}$-invariant prime divisors consist only of $V_{j}$ 's. This proves Theorem 1.1 (1) for the case when $\operatorname{dim} Y_{0}=0$.

Now we prove Theorem 1.1 (4) for the case when $\operatorname{dim} Y_{0}=0$. Since $s_{i}=n+\rho\left(X_{i}\right)$ for each $i$, the exceptional divisor of $u_{i}$ is contained in $\sum_{j} V(i)_{j}$ if $u_{i}$ is a divisorial contraction. Also, since $X_{r}$ and also $X$ are rationally connected with at worst klt singularities, by Proposition 3.1, $\operatorname{Pic}^{\circ}\left(X_{r}\right)=\operatorname{Pic}^{\circ}(X)=0$. Hence, in this case, Theorem 1.1 (4) follows from Lemma 3.5.

3.2.3. The Proof of Theorem 1.1 (1) for the Case $\operatorname{dim} Y_{0} \geq 1$. We still assume that $\operatorname{dim} Y_{0} \geq 1$ and $c \geq \rho(X)+\operatorname{dim} X$. Actually, the second part of (1) follows immediately. Indeed, if $c \geq 1$, then the ramification divisor $R_{f}>0$, which means $f$ is not étale in codimension one. By Lemma 2.22, $K_{X}$ is not pseudo-effective and hence $X$ is uniruled by Remark 2.8. Moreover, by Lemma 2.13, the pair $\left(X, \sum V_{j}\right)$ is $\log$ canonical.

Recall that Lemma 3.4 gives us the same inequality for each $X_{i}(i \leq r): s_{i} \geq n+\rho\left(X_{i}\right)$ if we assume $s_{0} \geq n+\rho\left(X_{0}\right)$. We want to ask whether the inequality still holds for $Y_{0}$. If the inequality holds, then we can use the induction on $\operatorname{dim} Y_{0}$, which proves Theorem 1.1 (1). Parallel to Lemma 3.4, we introduce the following lemma, from which, the first part of Theorem 1.1 follows immediately. 
To emphasize the notation for the Fano contraction $u_{r}: X_{r} \rightarrow Y_{0}$, let $s_{Y_{0}}$ be cardinality of $\mathrm{TI}_{g_{Y_{0}}}\left(Y_{0}\right)$ and $g_{Y_{0}}:=g_{r+1}$ the int-amplified endomorphism of $Y_{0}$, which $g_{0}$ descends to.

Lemma 3.7. With the same symbols given above, for the Fano contraction $u_{r}: X_{r} \rightarrow Y_{0}$, the inequality $s_{r} \leq s_{Y_{0}}+\operatorname{dim} X_{r}-\operatorname{dim} Y_{0}+1$ holds. Further, if $s_{r} \geq n+\rho\left(X_{r}\right)-k$ for some fixed integer $k$, then $s_{Y_{0}} \geq \operatorname{dim} Y_{0}+\rho\left(Y_{0}\right)-k$. In particular, if $k=0$, then $s_{Y_{0}}=\operatorname{dim} Y_{0}+\rho\left(Y_{0}\right)$ and also $s_{r}=n+\rho\left(X_{r}\right)$.

To prove Lemma 3.7, we need to do some preparations. By Lemma 2.7, the periodic points of $g_{Y_{0}}$ are dense. Let $y_{0} \in Y_{0}$ be a general $g_{Y_{0}}$-periodic point. Replacing $g_{Y_{0}}$ (and $\left.g_{i}\right)$ by its power, we may assume $g_{Y_{0}}\left(y_{0}\right)=y_{0}$. Let $W_{0}:=u_{r}^{-1}\left(y_{0}\right) \subseteq X_{r}$. Then $W_{0}$, as a general fibre of $u_{r}$, is a Fano variety of dimension $n-\operatorname{dim} Y_{0} \geq 1$, since the canonical divisor of a general fibre of a Fano contraction is anti-ample. Restricting $g_{r}$ to $W_{0}$, we get a surjective endomorphism of $W_{0}$ commuting with $u_{r}$ and $g_{Y_{0}}$ :

$$
g_{W_{0}}:=\left.g_{r}\right|_{W_{0}}: W_{0} \rightarrow W_{0} .
$$

Claim 3.8. With the same symbols given above, $g_{W_{0}}$ is polarized.

Proof. Since $u_{r}: X_{r} \rightarrow Y_{0}$ is a Fano contraction, the relative Picard number $\rho\left(X_{r} / Y_{0}\right)=$ 1. Therefore, pulling back a fixed ample divisor $H_{r}$ on $X_{r}$, we get

$$
g_{r}^{*} H_{r} \equiv k H_{r} \quad \bmod \left(u_{r}^{*} \mathrm{~N}^{1}\left(Y_{0}\right)\right) .
$$

Since $g_{r}$ is int-amplified, each eigenvalue of $\left.g_{r}^{*}\right|_{\mathrm{N}^{1}\left(X_{r}\right)}$ is of modulus greater than 1 (cf. Lemma 2.6). Besides, the operation $\left.g_{r}^{*}\right|_{\mathrm{N}^{1}\left(X_{r}\right)}$ induces an operation on the space $\mathrm{N}^{1}\left(X_{r}\right) / u_{r}^{*} \mathrm{~N}^{1}\left(Y_{0}\right)$, with all the eigenvalues of modulus greater than 1 . Thus, $k>1$.

Now, since $\left.u_{r}^{*} D\right|_{W_{0}}=0$ for any Cartier divisor $D$ on $Y_{0}$, restricting Equation (14) to a general fibre $W_{0}$, we get $\left.g_{W_{0}}^{*}\left(\left.H_{r}\right|_{W_{0}}\right) \equiv k H_{r}\right|_{W_{0}}$, with $\left.H_{r}\right|_{W_{0}}$ ample on $W_{0}$. This together with $k>1$ proves that $g_{W_{0}}$ is polarized (cf. [28, Lemma 2.3]).

Now, we begin to prove Lemma 3.7 .

Proof. We divide these $V(r)_{i}\left(1 \leq i \leq s_{r}\right)$ into two groups.

Case (1): Suppose $\left.u_{r}\right|_{V(r)_{j}}: V(r)_{j} \rightarrow Y_{0}\left(j \leq s_{r}(1)\right)$ is not surjective. Since $u_{r}$ is projective and thus closed, the image $u_{r}\left(V(r)_{j}\right)$ is a codimension $\geq 1$ closed subset. Take a general point $y_{0} \in Y_{0}$ such that $y_{0}$ does not lie in the image of any $V(r)_{j}$ for $j \leq s_{r}(1)$. Then one may easily get $\left.V(r)_{j}\right|_{W_{0}}=0$. Since $\operatorname{Pic}\left(X_{r} / Y_{0}\right)=1$, any two contracted curves are proportional (under the numerical equivalence). Hence, for any curve $C$ lying in a fibre of $X_{r} \rightarrow Y_{0},\left(V(r)_{j} \cdot C\right)=0$. Since $V(r)_{j}$ is $\mathbb{Q}$-Cartier, we may take a suitable $k_{j}^{\prime} \in \mathbb{N}$ such that $k_{j}^{\prime} V(r)_{j}$ is Cartier. 
By Cone Theorem (cf. [14, Lemma 3-2-5] or [17, Theorem $3.7(4)]), k_{j}^{\prime} V(r)_{j}=u_{r}^{*} G_{j}^{\prime}$ for some effective Cartier divisor $G_{j}^{\prime}$ on $Y_{0}$. Then the image $G_{j}:=u_{r}\left(V(r)_{j}\right)$, which is the support of $G_{j}^{\prime}$, is a prime divisor on $Y_{0}$. Since $Y_{0}$ is normal, on the smooth locus of $G_{j}$, the pullback of $G_{j}$ is an integral divisor. Taking the closure, we get $u_{r}^{*} G_{j}=k_{j} V(r)_{j}$ for some $k_{j} \in \mathbb{N}$. Moreover, by the commutative diagram, $g_{Y_{0}}^{-1}\left(G_{j}\right)=G_{j}$ set-theoretically for each $j \leq s_{r}(1)$. Applying the inductive hypothesis on $\operatorname{dim} Y_{0}$, the following holds:

$$
s_{r}(1) \leq s_{Y_{0}} \leq \operatorname{dim} Y_{0}+\rho\left(Y_{0}\right)
$$

Here, the case when $s_{r}(1)=0$ is allowed during our discussion.

Case (2): Suppose $\left.u_{r}\right|_{V(r)_{j}}: V(r)_{j} \rightarrow Y_{0}\left(s_{r}(1)<j \leq s_{r}\right)$ is surjective. Note that the general fibre $W_{0}$ is not contained in any $V(r)_{j}$ for $s_{r}(1)<j \leq s_{r}$. Let $s_{r}(2):=s_{r}-s_{r}(1)$ and fix an ample divisor $H_{X_{r}}$ on $X_{r}$, which does not lie in $u_{r}^{*} \mathrm{~N}^{1}\left(Y_{0}\right)$. Since $\rho\left(X_{r} / Y_{0}\right)=1$, we have the following numerical property:

$$
V(r)_{j} \equiv t_{j} H_{X_{r}} \bmod \left(u_{r}^{*} \mathrm{~N}^{1}\left(Y_{0}\right)\right)
$$

for some $t_{j}>0$, and thus $\left.V(r)_{j}\right|_{W_{0}}$ is ample on $W_{0}$. Moreover, $\left.V(r)_{j}\right|_{W_{0}}$ is $g_{W_{0}}^{-1}$-invariant on $W_{0}$ for each $s_{r}(1)<j \leq s_{r}$. Note that we also allow the case $s_{r}(2)=0$.

By Claim 3.8, $g_{W_{0}}$ is polarized. If $\operatorname{dim} W_{0} \geq 2$, then by Proposition 2.17, we get $s_{r}(2) \leq \operatorname{dim} W_{0}+1$; if $\operatorname{dim} W_{0}=1$, then by Lemma 3.3, we get $s_{r}(2) \leq 2=\operatorname{dim} W_{0}+1$. In any case, we get the following inequality:

$$
s_{r}(2) \leq \operatorname{dim} W_{0}+1 \text {. }
$$

Combining the inequality (16) with the first inequality of (15), the following holds:

(17) $s_{r}=s_{r}(1)+s_{r}(2) \leq s_{Y_{0}}+s_{r}(2) \leq s_{Y_{0}}+\operatorname{dim} W_{0}+1=s_{Y_{0}}+\operatorname{dim} X_{r}-\operatorname{dim} Y_{0}+1$

This completes the first part of our lemma.

If $s_{r} \geq \operatorname{dim} X_{r}+\rho\left(X_{r}\right)-k$ for some integer $k$, then by Equation (17) and the fact that $\rho\left(Y_{0}\right)=\rho\left(X_{r}\right)-1$, we have $s_{Y_{0}} \geq \operatorname{dim} Y_{0}+\rho\left(Y_{0}\right)-k$. In particular, if $k=0$, then combining (16) with the second inequality of (15), we see that,

$$
n+\rho\left(X_{r}\right) \leq s_{r}=s_{r}(1)+s_{r}(2) \leq \operatorname{dim} Y_{0}+\rho\left(Y_{0}\right)+\operatorname{dim} W_{0}+1=n+\rho\left(X_{r}\right) .
$$

Therefore, all the inequalities are equalities, and thus for each $i, s_{i}=n+\rho\left(X_{i}\right)$. This proves Lemma 3.7 and also Theorem 1.1 (1) for the case when $\operatorname{dim} Y_{0} \geq 1$ since $c \leq s_{0}$.

At the end of this part, the inductive hypothesis on $Y_{0}$ implies the following:

$$
s_{r}(1)=\operatorname{dim} Y_{0}+\rho\left(Y_{0}\right), K_{Y_{0}}+\sum_{i=1}^{s_{r}(1)} G_{i} \sim_{\mathbb{Q}} 0 .
$$


3.2.4. The Proof of Theorem 1.1 (2) and (3) for the Case When $\operatorname{dim} Y_{0} \geq 1$. In this part, we shall use Theorem 2.3 to prove Theorem $1.1(2),(3)$. Recall that we have such a $g_{0}$-equivariant relative MMP over $Y$

$$
X=X_{0}-->X_{1}-->\cdots-->X_{i}-->\cdots-->X_{r}-->X_{r+1}=Y_{0}-->Y .
$$

Here, we continue running MMP from $Y_{0}\left(X_{r} \rightarrow Y_{0}\right.$ is the first Fano contraction) and terminate with the end product $Y$. By the above discussion, we first consider the case when $Y=Y_{0}$, i.e. assume the MMP has only one Fano contraction. Suppose

$$
s_{0} \geq c \geq \operatorname{dim} X+\rho(X)-2 .
$$

Then by Lemmas 3.4 and 3.7, $s_{Y_{0}} \geq \operatorname{dim} Y_{0}+\rho\left(Y_{0}\right)-2$. Similarly, if $c \geq \operatorname{dim} X+\rho(X)-1$, then $s_{Y_{0}} \geq \operatorname{dim} Y_{0}+\rho\left(Y_{0}\right)-1$. Now we may start from $Y_{0}$ and continuously run MMP as mentioned in Theorem 2.3.

Claim 3.9. For the case when $s_{0} \geq \operatorname{dim} X+\rho(X)-2$, there are only two choices for the end product $Y$ of MMP: $Y$ is either an elliptic curve or a point.

Proof. Suppose the contrary that the claim does not hold. If $\operatorname{dim} Y \geq 2$, then $s_{Y} \geq$ $\operatorname{dim} Y+\rho(Y)-2 \geq 1$, which means the int-amplified endomorphism $g_{Y}$ has ramification divisors. Since $g_{Y}$ is not étale in codimension one, $K_{Y}$ is not pseudo-effective by Lemma 2.22. However, our end product $Y$ is $Q$-abelian and then $K_{Y} \sim_{\mathbb{Q}} 0$ (cf. Theorem 2.3), a contradiction. Therefore, $\operatorname{dim} Y=1$ and by Lemma 3.3, $Y$ is either elliptic or rational. If $Y \cong \mathbb{P}^{1}$, then $K_{Y}$ is not pseudo-effective. By Theorem 2.3 again, we can continue to contract $Y$ into a single point. This proves Claim 3.9.

The proof of Theorem 1.1 (3). If $Y$ is elliptic, then $s_{Y}=0$ (cf. Lemma 3.3), contradicting the deduced result $s_{Y} \geq \operatorname{dim} Y+\rho(Y)-1$. Therefore, $Y$ is a point (cf. Claim 3.9). By Theorem $2.3(2), X$ is rationally connected since the whole $X$ is a fibre. Then, by Proposition $3.1, \operatorname{Pic}^{\circ}(X)=0$ and then we may identify the Picard group with the Néron-Severi group. Further, $X$ is simply connected with respect to complex topology and $\operatorname{Pic}(X)$ is torsion free.

Theorem 2.3 asserts that $\left.g_{0}^{*}\right|_{\operatorname{Pic}(X)}$ is diagonalizable over $\mathbb{C}$ if and only if so is $g_{Y}^{*} \mid \operatorname{Pic}(Y)$. Since $Y$ is a point, $\left.g_{0}^{*}\right|_{\operatorname{Pic}(X)}$ is diagonalizable over $\mathbb{C}$. Furthermore, each eigenvalue $\lambda$ of $\left.g_{0}^{*}\right|_{\operatorname{Pic}(X)}$ is either an eigenvalue of $\left.g_{Y}^{*}\right|_{\operatorname{Pic}(Y)}$ or an eigenvalue of $\left.g_{i}^{*}\right|_{\operatorname{Pic}\left(X_{i}\right) / u_{i}^{*} \operatorname{Pic}\left(X_{i+1}\right)}$ for some $i$. Since $Y$ is a point, $\lambda$ is an eigenvalue of $\left.g_{i}^{*}\right|_{\operatorname{Pic}\left(X_{i}\right) / u_{i}^{*} \operatorname{Pic}\left(X_{i+1}\right)}$ for some $i$. Since $\rho\left(X_{i} / X_{i+1}\right)=1, \lambda \in \mathbb{Q}$. Indeed, all these eigenvalues are positive integers (cf. [24, Lemmas 5.1 and 5.2]). Thus, $\left.g_{0}^{*}\right|_{\operatorname{Pic}(X)}$ is diagonalizable over $\mathbb{Q}$. This completes the proof of Theorem $1.1(3)$. 
The proof of Theorem 1.1 (2). By Claim 3.9, there are two choices for the end product $Y$ of MMP. If $Y$ is a point, then similar to Theorem 1.1 (3), $X$ is rationally connected and simply connected with respect to complex topology; if $Y$ is an elliptic curve, then this $Y$ is our $E$ in Theorem 1.1 (2). By Theorem 2.3, $\phi: X \rightarrow Y=E$ is holomorphic and equi-dimensional with every fibre irreducible. Then $\phi: X \rightarrow E$ is proper and surjective, the general fibre of which is connected, and thus $\phi$ is a fibration. Further, $g_{0}=f^{t}$ descends to $g_{E}: E \rightarrow E$ of degree $>1$ (cf. Theorem 2.3). Furthermore, $\left.g_{0}^{*}\right|_{\mathrm{NS}_{\mathbb{Q}}(X)}$ is diagonalizable over $\mathbb{C}$ if and only if $\left.g_{E}^{*}\right|_{\mathrm{NS}_{\mathbb{Q}}(E)}$ is diagonalizable over $\mathbb{C}$ (cf. [23, Lemma 9.2]). Since we have proved $\left.g_{E}^{*}\right|_{\mathrm{NS}_{\mathbb{Q}}(E)}$ is diagonalizable over $\mathbb{Q}$ by Lemma 3.3, $\left.g_{0}^{*}\right|_{\mathrm{NS}_{\mathbb{Q}}(X)}$ is diagonalizable over $\mathbb{C}$. As in the case of Theorem $1.1(3)$, all the eigenvalues of $\left.g_{0}^{*}\right|_{\mathrm{NS}_{\mathbb{Q}}(X)}$ are rational numbers. Thus, $\left.g_{0}^{*}\right|_{\mathrm{NS}_{\mathbb{Q}}(X)}$ is diagonalizable over $\mathbb{Q}$.

Finally, we prove that every fibre of $\phi: X \rightarrow E$ is normal (cf. [32, The proof of Theorem 1.3]). Let $\Sigma=\left\{e \in E \mid \phi^{*} e=X_{e}\right.$ is not normal $\}$, a finite subset of $E$. By [28, The proof of Lemma 4.7], $g_{E}^{-1}(\Sigma) \subseteq \Sigma$, which implies $g_{E}^{-1}(\Sigma)=\Sigma$ since $\Sigma$ is a finite set. However, $g_{E}$ is étale and it could not have any $g_{E}^{-1}$-invariant divisors (cf. Lemma 3.3). As a result, $\Sigma=\emptyset$ and Theorem 1.1 (2) holds.

3.2.5. The Proof of Theorem 1.1 (4) for the case $\operatorname{dim} Y_{0} \geq 1$. We shall prove $K_{X}+\sum_{j=1}^{n+\rho} V_{j} \sim_{\mathbb{Q}} 0$ and $f$ is étale outside $f^{-1}(\operatorname{Sing} X) \cup\left(\cup V_{j}\right)$ for the case when $c=\operatorname{dim} X+\rho(X)$. In this part, the first Fano contraction is enough for our proof. As we proved in Subsubsection 3.2.3, if $s_{0} \geq c \geq \operatorname{dim} X+\rho(X)$, then $s_{0}=c=\operatorname{dim} X+\rho(X)$ and $s_{r}(2)=\operatorname{dim} W_{0}+1$, where $W_{0}$ is a general fibre of $X_{r} \rightarrow Y_{0}$.

Claim 3.10. Suppose $c=\operatorname{dim} X+\rho(X)$. Then $K_{W_{0}}+\left.\sum_{j=1}^{s_{r}} V(r)_{j}\right|_{W_{0}} \equiv 0$.

Proof. As we proved in Lemma 3.7, $\left.V(r)_{j}\right|_{W_{0}}$ is ample on $W_{0}$ for each $j>s_{r}(1)$. When $c=\operatorname{dim} X+\rho(X), s_{r}(2)=\operatorname{dim} W_{0}+1$. Besides, by Claim 3.8, $g_{W_{0}}=\left.g_{r}\right|_{W_{0}}$ is polarized by the restriction of an ample divisor on $X_{r}$. If $\operatorname{dim} W_{0}=1$, then by Case (b) of Lemma 3.3, $K_{W_{0}}+\left.\sum V(r)_{j}\right|_{W_{0}} \equiv 0$; if $\operatorname{dim} W_{0} \geq 2$, then applying Proposition 2.17 to the pair $\left(W_{0}, g_{W_{0}}\right)$ with $g_{W_{0}}^{-1}$-invariant ample divisors $\left.V_{j}\right|_{W_{0}}\left(j>s_{r}(1)\right)$, we have the following:

$$
\left.\left(K_{X_{r}}+\sum_{j=1}^{s_{r}} V(r)_{j}\right)\right|_{W_{0}} \equiv K_{W_{0}}+\left.\sum_{j=s_{r}(1)+1}^{s_{r}(1)+s_{r}(2)} V(r)_{l}\right|_{W_{0}} \equiv 0 .
$$

Here, $\left.V(r)_{j}\right|_{W_{0}}=0$ when $j \leq s_{r}(1)$ (cf. Case (1) in the proof of Lemma 3.7). We have completed the proof of our claim.

Recall Equation (8) for the case when the index $i=r$ as follows:

$$
K_{X_{r}}+\sum_{j=1}^{s_{r}} V(r)_{j}=g_{r}^{*}\left(K_{X_{r}}+\sum_{j=1}^{s_{r}} V(r)_{j}\right)+\Delta(r) .
$$


Restricting Equation (20) to the general fibre $W_{0}$ and then comparing it with Equation (19), we get $\left.\Delta(r)\right|_{W_{0}} \equiv 0$. Since $\Delta(r)$ is effective in the log ramification divisor formula, by Cone Theorem, $\Delta(r)$ is the pullback of some effective $\mathbb{Q}$-divisor $\Delta_{Y_{0}} \subseteq Y_{0}$.

Claim 3.11. $\Delta_{Y_{0}}=0$, i.e. $\Delta(r)=0$.

Suppose Claim 3.11 holds for the time being. Then by Equation (20), we have

$$
K_{X_{r}}+\sum_{j=1}^{s_{r}} V(r)_{j}=g_{r}^{*}\left(K_{X_{r}}+\sum_{j=1}^{s_{r}} V(r)_{j}\right) .
$$

Suppose $K_{X_{r}}+\sum_{j} V(r)_{j} \not \equiv 0$. Then from Equation (21), 1 is an eigenvalue for the operator $\left.g_{r}^{*}\right|_{\mathrm{NS}_{\mathbb{Q}}\left(X_{r}\right)}$, a contradiction (cf. Lemma 2.6). This forces $K_{X_{r}}+\sum V(r)_{j} \equiv 0$ and thus $K_{X_{r}}+\sum V(r)_{j} \sim_{\mathbb{Q}} 0$, since $\operatorname{Pic}^{\circ}\left(X_{r}\right)=\operatorname{Pic}^{\circ}\left(X_{0}\right)=0$ (cf. Proposition 3.1 and the proof of Theorem $1.1(3))$. Then Theorem 1.1 (4) follows from Lemma 3.5.

Now, the only thing we need to do is to prove Claim 3.11.

Proof of Claim 3.11. We shall construct a generically finite and surjective morphism to $Y_{0}$. Let $W\left(s_{r}+1\right):=X_{r}$. We take four steps to prove the claim.

Step 1. First, let $\sigma\left(s_{r}\right): W\left(s_{r}\right) \rightarrow X_{r}$ be the normalization $W\left(s_{r}\right) \rightarrow V(r)_{s_{r}}$ followed by an inclusion map $i: V(r)_{s_{r}} \hookrightarrow X_{r}$. Then $\operatorname{dim} W\left(s_{r}\right)=\operatorname{dim} X_{r}-1$. It is easy to get the following claim by considering the commutative diagram and applying Lemma 2.5 .

Claim 3.12. With the same symbols given above, there exists an int-amplified endomorphism $g\left(s_{r}\right)$ of $W\left(s_{r}\right)$ such that $\sigma\left(s_{r}\right) \circ g\left(s_{r}\right)=g_{r} \circ \sigma\left(s_{r}\right)$.

We return back to Step 1 of our proof for Claim 3.11. Pulling back Equation (20) along the morphism $\sigma\left(s_{r}\right)$, we get the following (cf. Claim 3.12):

$$
K_{W\left(s_{r}\right)}+D\left(s_{r}\right):=\sigma\left(s_{r}\right)^{*}\left(K_{X_{r}}+\sum V(r)_{j}\right)=g\left(s_{r}\right)^{*}\left(K_{W\left(s_{r}\right)}+D\left(s_{r}\right)\right)+\Delta(r)_{s_{r}} .
$$

Here, $\Delta(r)_{s_{r}}$ is the pullback of $\Delta(r)$. Note that each $V(r)_{j}\left(j \leq s_{r}(1)\right)$ intersects $V(r)_{s_{r}}$ (cf. the proof of Lemma 3.7). Indeed, since $k_{j} V(r)_{j}=u_{r}^{*} G_{j}$ for each $j \leq s_{r}(1), V(r)_{j}$ contains all the fibres over $G_{j}$. Also, $V(r)_{s_{r}}$ dominates $Y_{0}$ and hence $V(r)_{j}$ must intersect $V(r)_{s_{r}}$. Then the pullback of each $V(r)_{j}\left(j \leq s_{r}(1)\right)$ survives in $W\left(s_{r}\right)$. The next claim follows from [30, Corollary 3.11] and [15, Corollary 16.7] (also cf. [19, Proposition 2.5]).

Claim 3.13. The pair $\left(W\left(s_{r}\right), D\left(s_{r}\right)\right)$ is log canonical and the support of the pullback $\sum_{j=1}^{s_{r}(1)} \sigma\left(s_{r}\right)^{*} V(r)_{j}$ (as a reduced divisor) is contained in $D\left(s_{r}\right)$, i.e.

$$
D\left(s_{r}\right) \geq \sum_{j=1}^{s_{r}(1)} \operatorname{Supp} \sigma\left(s_{r}\right)^{*} V(r)_{j}
$$


Proof. The first part follows from Proposition 2.1. For the second part, on the one hand, since $\left(W\left(s_{r}\right), D\left(s_{r}\right)\right)$ is log canonical, the coefficient of each component in $D\left(s_{r}\right)$ cannot exceed 1. On the other hand, for each irreducible component $P(j)_{i}$ of the support of the intersection $V(r)_{s_{r}} \cap V(r)_{j}$, note that $\left(X_{r}, V(r)_{s_{r}}\right)$ should be log terminal around $P(j)_{i}$ (cf. [17, Lemma 2.27]). Hence, the coefficient of $P(j)_{i}$ in $D\left(s_{r}\right)$ is given by (cf. [30, Corollaries 3.10 and 3.11])

$$
1-\frac{1}{m}+\sum \frac{r_{j}}{m}
$$

where $r_{j} / m$ is the multiplicity of $\sigma\left(s_{r}\right)^{*} V(r)_{j}$ around $P(j)_{i}$ and $r_{j}$ is a natural number. Since $V(r)_{j}\left(j \leq s_{r}(1)\right)$ intersects $V(r)_{s_{r}}$ as we discussed above, $r_{j} \geq 1$ and hence the coefficient of $P(j)_{i}$ in $D\left(s_{r}\right)$ is no less than 1 . In conclusion, the total coefficient of each component of $\operatorname{Supp} \sigma\left(s_{r}\right)^{*} V(r)_{j}\left(j \leq s_{r}(1)\right)$ in $D\left(s_{r}\right)$ is 1 and our claim holds.

We come back to the proof of Claim 3.11. Suppose $\operatorname{dim} X_{r}-\operatorname{dim} Y_{0}=1$. Then we go directly to Step 4 with $k_{0}=s_{r}$.

Step 2. Suppose $\operatorname{dim} X_{r}-\operatorname{dim} Y_{0} \geq 2$. Then $\operatorname{dim} W_{0} \geq 2$. Therefore, $\left.V(r)_{s_{r}}\right|_{W_{0}}$ is connected since it is ample on $W_{0}$ (cf. [10, Corollary 7.9]). Further, for each $s_{r}(1)<j<s_{r}$, $\left.V(r)_{j}\right|_{W_{0}}$ is also ample and thus nonzero on $W_{0}$.

Since $V(r)_{s_{r}}$ also intersects $V(r)_{j}$ for each $s_{r}(1)<j<s_{r}$ by the ampleness of $V(r)_{j}$. With the same proof of Claim 3.13, the support of $\sigma\left(s_{r}\right)^{*} V(r)_{j}\left(s_{r}(1)<j<s_{r}\right)$, which is a reduced divisor, is contained in $D\left(s_{r}\right)$.

We claim that each $\operatorname{Supp} \sigma\left(s_{r}\right)^{*} V(r)_{j}\left(s_{r}(1)<j<s_{r}\right)$ still dominates $Y_{0}$. Indeed, a general fibre of $V(r)_{s_{r}} \hookrightarrow X_{r} \rightarrow Y_{0}$ is as the form of $W_{0} \cap V(r)_{s_{r}}$. Since each $\left.V(r)_{j}\right|_{W_{0}}\left(s_{r}(1)<j<s_{r}\right)$ is ample on $W_{0}$, the restriction $\left.V(r)_{j}\right|_{W_{0} \cap V(r)_{s_{r}}}$ is still ample on $W_{0} \cap V(r)_{s_{r}}$ for $s_{r}(1)<j<s_{r}$. Note that this can only be obtained under the condition that $\operatorname{dim} W_{0} \geq 2$. Therefore, the nonzero divisor $\left.V(r)_{j}\right|_{V(r)_{s_{r}}}$ on $V(r)_{s_{r}}$ intersects the general fibre of $V(r)_{s_{r}} \rightarrow Y_{0}$ and thus its support dominates $Y_{0}$. Moreover, normalization is finite, and hence $\sigma\left(s_{r}\right)^{*} V(r)_{j}$ is nonzero and ample when restricted to any general fibre of $W\left(s_{r}\right) \rightarrow Y_{0}$ for any $s_{r}(1)<j<s_{r}$. As in the proof of Proposition 2.17, we fix an (integral) irreducible component $\operatorname{Supp} B_{s_{r}-1}\left(\leq D\left(s_{r}\right)\right)$ of $\operatorname{Supp} \sigma\left(s_{r}\right)^{*} V(r)_{s_{r}-1}$ dominating $Y_{0}$ and then take the normalization $W\left(s_{r}-1\right)$ of $B_{s_{r}-1}$ followed by the inclusion to get the next morphism $\sigma\left(s_{r}-1\right): W\left(s_{r}-1\right) \rightarrow W\left(s_{r}\right)$.

Step 3. In general, for each $s_{r} \geq k \geq s_{r}-\operatorname{dim} W_{0}+1=s_{r}(1)+2$, let $\sigma(k)$ : $W(k) \rightarrow W(k+1)$ be the normalization of a fixed (integral) irreducible component $B_{k}$ of Supp $\left.V(r)_{k}\right|_{W(k+1)}$ dominating $Y_{0}$, followed by inclusion. Such $B_{k}$ exists by the induction and the following condition. Since the dimension of the fibre of $W(k+2) \rightarrow Y_{0}$ is $\operatorname{dim} W_{0}-s_{r}+k+1$ which is at least 2 by assumption, $\left(\sigma\left(s_{r}\right) \circ \cdots \circ \sigma(k+2)\right)^{*} V(r)_{k}$ 
intersects $B_{k+1}$ and hence the support of the pullback $\left.V(r)_{k}\right|_{W(k+1)}$ dominates $Y_{0}$ with the same proof as in Step 2.

Moreover, for each $k \geq s_{r}(1)+2, B_{k}$ intersects $\left.V(r)_{j}\right|_{W(k+1)}\left(j \leq s_{r}(1)\right)$ and hence the support of the pullback of $V(r)_{j}\left(j \leq s_{r}(1)\right)$ to $W(k)$ (as a reduced divisor) is contained in $D(k)$ (cf. Claim 3.13). Now, $\operatorname{dim} W(k)=\operatorname{dim} X_{r}-\left(s_{r}-k\right)-1$ and similar to Claim 3.12, we get an int-amplified endomorphism $g(k)$ of $W(k)$, commuting with each $g(l)$ for $l>k$. Therefore, we get the following equations:

$$
K_{W(k)}+D(k):=\sigma(k)^{*}\left(K_{W(k+1)}+D(k+1)\right)=g(k)^{*}\left(K_{W(k)}+D(k)\right)+\Delta(r)_{k}
$$

with $k=s_{r}, s_{r-1}, \cdots, k_{0}:=s_{r}(1)+2$. For each $k, \Delta(r)_{k}$ is the pullback of $\Delta(r)$; the pair $(W(k), D(k))$ is $\log$ canonical with $D(k) \geq\left.\sum_{j=1}^{s_{r}(1)} \operatorname{Supp} V(r)_{j}\right|_{W(k)}$.

Step 4. Let $R_{j}\left(j \leq s_{r}(1)\right)$ denote the support of the pullback of $V(r)_{j}$ to $W\left(k_{0}\right)$, which are all reduced integral divisors (may not be irreducible). Then $D\left(k_{0}\right) \geq \sum_{j=1}^{s_{r}(1)} R_{j}$. Let

$$
\tau_{k_{0}}:=u_{r} \circ \sigma\left(s_{r}\right) \cdots \circ \sigma\left(k_{0}\right): W\left(k_{0}\right) \rightarrow \cdots \rightarrow X_{r} \rightarrow Y_{0}
$$

By construction, $\tau_{k_{0}}$ is dominant and projective. Further, $\operatorname{dim} W\left(k_{0}\right)=\operatorname{dim} Y_{0}$, and thus $\tau_{k_{0}}$ is generically finite. By the commutative diagram, we have $\tau_{k_{0}} \circ g\left(k_{0}\right)=g_{Y_{0}} \circ \tau_{k_{0}}$. Also, taking the Stein factorization of $\tau_{k_{0}}$, we get a birational morphism $\nu: W\left(k_{0}\right) \rightarrow S\left(k_{0}\right)$ with connected fibres and a finite morphism $\gamma: S\left(k_{0}\right) \rightarrow Y_{0}$ such that $\tau_{k_{0}}=\gamma \circ \nu$.

On the one hand, since $\gamma$ is finite, by the log ramification divisor formula, we have:

$$
K_{S\left(k_{0}\right)}+\sum \operatorname{Supp} \gamma^{*} G_{j}-R_{\gamma}=\gamma^{*}\left(K_{Y_{0}}+\sum G_{j}\right)
$$

where $R_{\gamma}$ is effective, having no common components with $\sum \operatorname{Supp} \gamma^{*} G_{j}$. Since the righthand side of Equation (23) is $\mathbb{Q}$-Cartier, we can pull back Equation (23) to $W\left(k_{0}\right)$ under the birational morphism $\nu$ (cf. [4, Lemma 2.7]):

$$
K_{W\left(k_{0}\right)}+\nu_{*}^{-1}\left(\sum_{j=1}^{s_{r}(1)} \operatorname{Supp} \gamma^{*} G_{j}-R_{\gamma}\right)-F=\tau_{k_{0}}^{*}\left(K_{Y_{0}}+\sum_{j=1}^{s_{r}(1)} G_{j}\right) .
$$

Here, $F$ is $\nu$-exceptional, which may not be effective. On the other hand, we recall the following equations (cf. Equation (22) and the proof of Lemma 3.7):

$$
\begin{aligned}
& K_{W\left(k_{0}\right)}+D\left(k_{0}\right)=g\left(k_{0}\right)^{*}\left(K_{W\left(k_{0}\right)}+D\left(k_{0}\right)\right)+\Delta(r)_{k_{0}}, \\
& k_{j} V(r)_{j}=u_{r}^{*} G_{j}\left(1 \leq j \leq s_{r}(1)\right), \Delta(r)=u_{r}^{*} \Delta_{Y_{0}} .
\end{aligned}
$$

Putting Equation (24), (25) and (26) together, we get

$$
\tau_{k_{0}}^{*}\left(K_{Y_{0}}+\sum G_{j}\right)+M+F=g\left(k_{0}\right)^{*}\left(\tau_{k_{0}}^{*}\left(K_{Y_{0}}+\sum G_{j}\right)+M+F\right)+\tau_{k_{0}}^{*} \Delta_{Y_{0}} .
$$


Here, $M:=D\left(k_{0}\right)-\nu_{*}^{-1}\left(\sum_{j=1}^{s_{r}(1)} \operatorname{Supp} \gamma^{*} G_{j}\right)+\nu_{*}^{-1} R_{\gamma}$ and we claim that $M$ is effective. Indeed, according to Equation (26) and the notation at the beginning of Step 4, we see that,

$$
\nu_{*}^{-1} \sum_{j=1}^{s_{r}(1)} \operatorname{Supp} \gamma^{*} G_{j} \leq \sum_{j=1}^{s_{r}(1)} \operatorname{Supp} \tau_{k_{0}}^{*} G_{j}=\sum_{j=1}^{s_{r}(1)} R_{j} .
$$

Therefore $M \geq D\left(k_{0}\right)-\sum R_{j}+\nu_{*}^{-1} R_{\gamma} \geq 0$ (cf. Claim 3.13), which completes the proof of our claim. Further, applying the inductive hypothesis on $Y_{0}$ (cf. Equation (18)) to Equation (27), we get

$$
M+F \equiv g\left(k_{0}\right)^{*}(M+F)+\tau_{k_{0}}^{*} \Delta_{Y_{0}} .
$$

Now, suppose that $\Delta_{Y_{0}} \neq 0$. Then $N:=\tau_{k_{0}}^{*} \Delta_{Y_{0}}$ is some nonzero effective $\mathbb{Q}$-divisor, which is not $\nu$-exceptional on $W\left(k_{0}\right)$. We shall apply the method in the proof of Lemma 2.20 to find the contradiction. Multiplying Equation (28) by a positive integer, we may assume $N$ is integral. Then substituting the expression of $M+F$ to the right-hand side $(m-1)$-times, we get

$$
M+F=\left(g\left(k_{0}\right)^{m}\right)^{*}(M+F)+\sum_{j=0}^{m-1}\left(g\left(k_{0}\right)^{j}\right)^{*} N .
$$

According to the commutative diagram, $\left(g\left(k_{0}\right)^{m}\right)^{*} F$ is also $\nu$-exceptional for each natural number $m$. Fixing an ample Cartier divisor $H$ on $S\left(k_{0}\right)$ and pulling back to $W\left(k_{0}\right)$ along the birational morphism $\nu$, we get the following inequality by projection formula:

$$
\left(M \cdot \nu^{*} H^{\operatorname{dim} W\left(k_{0}\right)-1}\right) \geq \sum_{j=0}^{m-1}\left(\nu_{*}\left(g\left(k_{0}\right)^{j}\right)^{*} N \cdot H^{\operatorname{dim} S\left(k_{0}\right)-1}\right) .
$$

Note that $N$ is integral and not $\nu$-exceptional, and thus $\nu_{*}\left(g\left(k_{0}\right)^{j}\right)^{*} N$ is an effective integral divisor on $S\left(k_{0}\right)$. So the right-hand side tends to infinity if we let $m \rightarrow \infty$, a contradiction. This in turn completes the proof of Claim 3.11 and also Theorem 1.1 (4).

We give the following remark at the end of the proof of Theorem 1.1.

Remark 3.14. When extending the results in [32, Theorem 1.3], we improve the original proof from the following points. First, when considering the Fano contraction $u_{r}: X_{r} \rightarrow$ $Y_{0}$, the pullback of $G_{j}\left(j \leq s_{r}(1)\right)$ is a multiple of $V(r)_{j}$ and thus may not be reduced, since $V(r)_{j}$ is only $\mathbb{Q}$-Cartier; Second, when proving Proposition 2.17, $D_{i}$ may not be reduced while it holds for the case when $s=n+1$ (cf. the proof of Remark 2.21 and [32, Proposition 2.12]); Finally, for each $V(r)_{j}$, the restriction to general fibre $W_{0}$ may be reducible (but still reduced) even though $V(r)_{j}$ is irreducible, and thus we need to extend the result of [Ibid.] to the case when $V_{j}$ are reduced (cf. Proposition 2.17). 


\section{REFERENCES}

[1] P. Berthelot et al, Theorie des Intersections et Theoreme de Riemann-Roch: Seminaire de Geometrie Algebrique du Bois Marie 1966/67 (SGA 6), Lecture Notes Math., Vol. 225, Springer, New York (2006).

[2] S. Boucksom, et al.: The pseudo-effective cone of a compact Kähler manifold and varieties of negative Kodaira dimension, J. Algebr. Geom., Vol. 22(2013), no. 2, 201-248, arXiv:0405285.

[3] M. Brown, J. McKernan, R. Svaldi, and H. Zong, A geometric characterisation of toric varieties, Duke Math. J., Vol. 167(2018), no. 5, 923-968, arXiv:1605.08911.

[4] A. Broustet and A. Höring, Singularities of varieties admitting an endomorphism, Math. Ann., Vol. 360(2014), no. 1-2, 439-456.

[5] F. Campana, On twistor spaces of the class $\mathscr{C}$, J. Differ. Geom., Vol. 33(1991), no. 2, 541-549.

[6] P. Cascini, S. Meng and D.-Q. Zhang, Polarized endomorphisms of normal projective threefolds in arbitrary characteristic, Math. Ann. (to appear), arXiv:1710.01903, 2017.

[7] N. Fakhruddin, Questions on self maps of algebraic varieties, J. Ramanujan Math. Soc., Vol. 18(2003), no. 2, 109122, arXiv:math/0212208.

[8] M. Fulger, J. Kollár, and B. Lehmann, Volume and Hilbert function of $\mathbb{R}$-divisors, Mich. Math. J., Vol. 65(2016), no. 2, 371-387.

[9] W. Fulton, Intersection Theory, Ergeb. Math. Grenzgeb. (3), Vol. 2, Springer-Verlag, 1984.

[10] R. Hartshorne, Algebraic Geometry, Graduate Texts Math., Vol. 52, New York: Springer-Verlag, 2013.

[11] A. Hatcher, Algebraic Topology, Cambridge: Cambridge University Press, 2000.

[12] S. Iitaka, Algebraic Geometry-An introduction to Birational Geometry of Varieties, Graduate Texts Math., Vol. 76, New York: Springer-Verlag, 1982.

[13] M. Kawakita, Inversion of adjunction on log canonicity, Invent. Math., Vol. 167(2007), no. 1, 129133, arXiv:0511254.

[14] Y. Kawamata, K. Matsuda and K. Matsuki, Introduction to the minimal model problem, Adv. Stud. Pure Math., Vol. 10(1987), 283-360.

[15] J. Kollár et al, Flips and Abundance for Algebraic Threefolds, Lectures in Utah Summer Seminar 1991, Asterisque, Vol. 211, Societe Mathematique de France, Paris (1992).

[16] J. Kollár, Y. Miyaoka and S. Mori, Rational connectedness and boundedness of fano manifolds, J. Differ. Geom., Vol. 36(1992), no.3, 765-779.

[17] J. Kollár and S. Mori, Birational geometry of algebraic varieties, Cambridge Tracts in Math., Vol. 134, Cambridge: Cambridge Univ. Press, 1998.

[18] J. Kollár, Rational curves on algebraic varieties, Ergeb. Math. Grenzgeb. (3), Vol. 32, New York: Springer-Verlag, 1999.

[19] J. Kollár, Singularities of the minimal model program, Cambridge Tracts in Mathematics, Vol. 200, With a collaboration of Sándor Kovács, Cambridge University Press, Cambridge, 2013.

[20] H. Krieger and P. Reschke, Cohomological conditions on endomorphisms of projective varieties, Bull. Soc. Math. Fr., Vol. 145(2017), no.3, 449-468, arXiv:1505.07088.

[21] R.K. Lazarsfeld, Positivity in Algebraic Geometry I: Classical Setting: Line Bundles and Linear Series, Ergeb. Math. Grenzgeb. (3), Vol. 48, Springer, New York (2004). 
[22] S. Meng, Building blocks of amplified endomorphisms of normal projective varieties, Math. Z. (to appear), arXiv:1712.08995, 2017.

[23] S. Meng and D.-Q. Zhang, Building blocks of polarized endomorphisms of normal projective varieties, Adv. Math., Vol. 325(2018), 243-273, arXiv:1606.01345v4.

[24] S. Meng and D.-Q. Zhang, Semi-group structure of all endomorphisms of a projective variety admitting a polarized endomorphism, Math. Res. Lett. (to appear), arXiv:1806.05828, 2018.

[25] D. Mumford, Abelian Varieties, With Appendices by C. P. Ramanujam and Yuri Manin, Corrected Reprint of the Second (1974) Edition, Tata Institute of Fundamental Research Studies in Mathematics, Vol. 5, Published for the Tata Institute of Fundamental Research, Bombay, 2008.

[26] N. Nakayama, Zariski-Decomposition and Abundance, MSJ Mem., Vol. 14, Jpn, Tokyo (2004).

[27] N. Nakayama, On complex normal projective surfaces admitting non-isomorphic surjective endomorphisms, Preprint 2 September 2008.

[28] N. Nakayama and D.-Q. Zhang, Polarized endomorphisms of complex normal varieties, Math. Ann., Vol. 346(2010), no.4, 991-1018, arXiv:0909.1688v1.

[29] B. Poonen, Rational Points on Varieties, Graduate Studies in Mathematics, Vol. 186, American Mathmatical Society, Providence (2017).

[30] V.V. Shokurov, 3-fold log flips, Izn. A. N. Ser. Mat. Vol. 56(1992), 105-203 (in Russian).

[31] S. Takayama, Local simple connectedness of resolutions of log-terminal singularities, Int. J. Math., Vol. 14(2003), no.8, 825-836.

[32] D.-Q. Zhang, Invariant hypersurfaces of endomorphisms of projective varieties, Adv. Math., Vol. 252(2014), 185-203, arXiv:1310.5944.

[33] D.-Q. Zhang, $n$-dimensional projective varieties with the action of an abelian group of rank $n-1$, Trans. Amer. Math. Soc., Vol. 368(2016), no.12, 8849-8872. arXiv:1412.5779.

[34] Q. Zhang, Rational connectedness of $\log q$-fano varieties, J. Reine Angew. Math., Vol. 590(2006), 131-142, arXiv:0408301.

Department of Mathematics

National University of Singapore, Singapore 119076, Republic of Singapore

E-mail address: zhongguolei@u.nus.edu 